\title{
Deep Learning Techniques for the Classification of Colorectal Cancer Tissue
}

\author{
Min-Jen Tsai * ${ }^{-1}$ and Yu-Han Tao
}

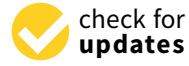

Citation: Tsai, M.-J.; Tao, Y.-H. Deep Learning Techniques for the Classification of Colorectal Cancer Tissue. Electronics 2021, 10, 1662. https://doi.org/10.3390/ electronics10141662

Academic Editor: Tadeusz A. Wysocki

Received: 31 May 2021

Accepted: 8 July 2021

Published: 12 July 2021

Publisher's Note: MDPI stays neutral with regard to jurisdictional claims in published maps and institutional affiliations.

Copyright: (c) 2021 by the authors. Licensee MDPI, Basel, Switzerland. This article is an open access article distributed under the terms and conditions of the Creative Commons Attribution (CC BY) license (https:/ / creativecommons.org/licenses/by/ $4.0 /)$.
Institute of Information Management, National Yang Ming Chiao Tung University, Hsin-Chu 300, Taiwan; mjtsai@iim.nctu.edu.tw

* Correspondence: mjtsai@cc.nctu.edu.tw

Abstract: It is very important to make an objective evaluation of colorectal cancer histological images. Current approaches are generally based on the use of different combinations of textual features and classifiers to assess the classification performance, or transfer learning to classify different organizational types. However, since histological images contain multiple tissue types and characteristics, classification is still challenging. In this study, we proposed the best classification methodology based on the selected optimizer and modified the parameters of CNN methods. Then, we used deep learning technology to distinguish between healthy and diseased large intestine tissues. Firstly, we trained a neural network and compared the network architecture optimizers. Secondly, we modified the parameters of the network layer to optimize the superior architecture. Finally, we compared our well-trained deep learning methods on two different histological image open datasets, which comprised $5000 \mathrm{H} \& \mathrm{E}$ images of colorectal cancer. The other dataset was composed of nine organizational categories of 100,000 images with an external validation of 7180 images. The results showed that the accuracy of the recognition of histopathological images was significantly better than that of existing methods. Therefore, this method is expected to have great potential to assist physicians to make clinical diagnoses and reduce the number of disparate assessments based on the use of artificial intelligence to classify colorectal cancer tissue.

Keywords: convolutional neural network; machine learning; deep learning; colorectal cancer

\section{Introduction}

Colorectal cancer (CRC) is the third most common form of cancer, accounting for about $10 \%$ of all cases in the world [1]. The results of many studies have shown that a more accurate classification of medical images can effectively determine the development of colorectal cancer [2,3]. Many common tissue types, such as normal colon mucosa (NORM), adipose tissue (ADI), polyps, cancer-associated stroma (STR), and lymphocytes (LYM) can extract prognosticators directly from these hematoxylin and eosin stains (HE stains), which are the principal tissue stains used in histology [2]. Optical colonoscopy is the medical procedure that is usually used to examine a series of abnormalities on the surface of the colon, including their location, morphology and pathological changes to make a clinical diagnosis. This improves the accuracy of the diagnosis and the ability to predict the severity of the disease in order to apply the most appropriate clinical treatment. Nevertheless, although the correct classification of pathological images is an important factor in assisting doctors to precisely identify the best possible treatment, a great deal of time and effort is required to analyze histopathological images, and the evaluation of tissue classification is easily affected by many subjective factors. Subjective evaluation is generally performed by pathologists who manually review the histological slides images of CRC tissue, which remains the standard for cancer diagnosis and staging. However, the training, experience, evaluation condition or time pressure for each pathologist could result different diagnosis judgement. Hence, the universal automatic classification of CRC pathological tissue slide images for fair evaluation has an important clinical significance. 
Pathology slides provide an enormous amount of information, which has been quantified through digital pathology and classic machine learning techniques over the years [4]. Previous research has been based on machine learning approaches for judging the cell classification in the histological slides of tumor tissue. The classification of histopathological images using artificial intelligence not only improves the accuracy and efficiency of the classification, but also enables doctors to make timely decisions in terms of clinical treatment $[5,6]$. However, most of the proposed experimental methods rely on manual feature labels, which is the main limitation of traditional textual analysis approaches. Therefore, deep learning has been introduced in the last few years to solve this and other limitations. Deep learning is a new technology that is considered to be an evolution of machine learning, since it uses multiple layers of neural networks to learn and progressively extract higher-level features in order to reduce human intervention in the recognition of different classes in the images. It is also effective in classifying non-image data, such as speech recognition, social network filtering, and medical image analysis, and its advanced approach not only reduces the need for human intervention, but it can also automatically achieve results that are comparable to or surpassing those of humans.

Convolutional neural networks $(\mathrm{CNN})[7,8]$ recently showed effective results in classifying images in the field of deep learning where a neural network might have dozens or hundreds of layers to learn containing images with different features. A convolutional layer composed of a small-sized kernel to generate advanced features applies weights to the inputs and directs them through an activation function as the output. The main advantage of using CNN compared to a traditional neural network is that it reduces the model parameters for more accurate results.

With this in mind, we aimed to use deep learning technology to identify medical images to increase the accuracy of the identification due to the automatic classification of tumor types. This involved the achievement of the following objectives:

a. To compare the classification accuracy rate with different CNN models.

b. To find the best performance of deep learning techniques.

c. To compare the results of this method with those of existing techniques.

This paper consists of a systematic study of deep learning and its application for the classification of pathological images. Past studies of deep learning will be reviewed in Section 2, while the approach of deep learning models will be described in Section 3. Details of the experiment will be provided in Section 4, and the paper will be concluded in Section 5 with proposals for possible future investigations in this field.

\section{Related Works and Deep Learning Methodology}

Some of the prior studies in relation to the automatic classification of histopathological images will be described and discussed in this section with a further explanation of how deep learning works. This will be followed by a presentation of the proposed method to conduct the current research.

\subsection{Related Works}

Digital technology is currently used extensively to classify medical images, as evidenced by the results of several methods of histopathological image classification shown in Table 1. Kather [2] used a range of textual descriptors to analyze a multi-class problem of tumor epithelium and simple stroma in 5000 histological images. He proposed four classification methods: (1) the k-nearest neighbors algorithm (k-NN), (2) employ an SVM decision function in an attempt to classify all categories, (3) assemble decision tree models using the RUSBoost method, and (4) use a 10-fold cross validation to train the classifiers, without an explicit stratification approach. The results indicated that SVM was the best classification method, which achieved $87.4 \%$ accuracy over eight classes. Lately, the classification of tumor types has been found to be more accurate using the $\mathrm{CNN}$ classification method. Tsai [9] applied the CNN architecture of a deep learning technique to detect pneumonia 
from Chest $\mathrm{X}$-rays and achieved an accuracy rate within $82.1 \%$ by using feature selection and the CNN.

Table 1. Related Research.

\begin{tabular}{|c|c|c|c|c|}
\hline Literature & Research Objective & Approach & $\begin{array}{l}\text { Classification } \\
\text { Technique }\end{array}$ & Accuracy Rate (\%) \\
\hline [2] & $\begin{array}{l}\text { Multi-class texture } \\
\text { analysis in colorectal } \\
\text { cancer histology }\end{array}$ & Texture based methods & $\begin{array}{l}\text { One-nearest neighbor, } \\
\text { linear SVM, } \\
\text { radial-basis function } \\
\text { SVM and decision } \\
\text { trees. }\end{array}$ & 87.4 \\
\hline [9] & $\begin{array}{c}\text { Machine } \\
\text { learning-based } \\
\text { common } \\
\text { radiologist-level } \\
\text { pneumonia detection } \\
\text { on chest X-rays }\end{array}$ & Feature selection, $\mathrm{CNN}$ & $\begin{array}{l}\text { Feature selection and } \\
\text { convolutional neural } \\
\text { network }(\mathrm{CNN})\end{array}$ & 80.9 \\
\hline [10] & $\begin{array}{l}\text { A deep convolutional } \\
\text { neural network for } \\
\text { segmenting and } \\
\text { classifying the } \\
\text { epithelial and stromal } \\
\text { regions in } \\
\text { histopathological } \\
\text { images }\end{array}$ & $\mathrm{CNN}$ & $\begin{array}{c}\text { CNN network } \\
\text { comprised of two } \\
\text { convolutional layers, } \\
\text { two max-pooling } \\
\text { layers, and two fully } \\
\text { connected layers } \\
\text { followed by a soft-max } \\
\text { layer }\end{array}$ & 84 \\
\hline [11] & $\begin{array}{l}\text { Classification of tumor } \\
\text { epithelium and stroma } \\
\text { by exploiting image } \\
\text { features learned by } \\
\text { deep convolutional } \\
\text { neural networks }\end{array}$ & $\mathrm{CNN}$ & $\begin{array}{c}\text { CNN with GoogLeNet } \\
\text { transfer learning } \\
\text { strategies }\end{array}$ & 90.2 \\
\hline [12] & $\begin{array}{c}\text { Large-scale tissue } \\
\text { histopathology image } \\
\text { classification, } \\
\text { segmentation, and } \\
\text { visualization via deep } \\
\text { convolutional } \\
\text { activation features }\end{array}$ & $\mathrm{CNN}$ & $\begin{array}{l}\text { The framework transfer } \\
\text { features extracted from } \\
\text { CNN were trained by a } \\
\text { large natural image } \\
\text { database, ImageNet, to } \\
\text { assess the } \\
\text { histopathology images } \\
\text { and also explore the } \\
\text { characteristics in the } \\
\text { last hidden layer }\end{array}$ & 97.5 \\
\hline [13] & $\begin{array}{c}\text { Using deep } \\
\text { convolutional neural } \\
\text { networks to identify } \\
\text { and classify } \\
\text { tumor-associated } \\
\text { stroma in diagnostic } \\
\text { breast biopsies }\end{array}$ & $\mathrm{CNN}$ & $\begin{array}{l}\text { Propose some new } \\
\text { geometric features of } \\
\text { benign biopsies }\end{array}$ & 96.2 \\
\hline [3] & $\begin{array}{l}\text { Predicting survival } \\
\text { from colorectal cancer } \\
\text { histology slides using } \\
\text { deep learning, a } \\
\text { retrospective } \\
\text { multicenter study }\end{array}$ & $\mathrm{CNN}$ & $\begin{array}{c}\text { Evaluated the } \\
\text { performance of five } \\
\text { different CNN models: } \\
\text { VGG19, AlexNet, } \\
\text { SqueezeNet, } \\
\text { GoogLeNet, Resnet50 }\end{array}$ & 98.7 \\
\hline
\end{tabular}

$\mathrm{Xu}$ [10] used the CNN model and feature extraction approaches to compare two datasets of breast cancer and colorectal cancer. The two types of tissues in the histological 
images were epithelial (EP) and stromal (ST). He used automated segmentation or the classification of color features, which included intensive pixels in different color spaces, and analyzed the tumor microenvironment. In his study, Du [11] proposed that learning the basic features of CNN methods outperformed handcrafted features, and automatically distinguished the epithelial and stromal regions in the breast. In addition, he found that colorectal tumors could be distinguished from tumor tissue using a network architecture layer approach with results that were $84 \%$ accurate. Transfer learning is a methodology that consists of deep learning techniques to distinguish the features of leverages images. $\mathrm{Du}$ [10] discussed the use of transfer learning methods to accurately distinguish breast or ovarian cancer from histological images and of CNN for fine tuning the feature extractor of images. Additionally, he discussed how to distinguish high-level and low-level features inside the neural network. A deep neural network may have multiple layers, the first of which will learn the low-level features and then the more they progress toward the output layer, the more the layers will learn the high-level features. Du [11] also used a transfer learning approach with GoogLeNet and achieved 90.2\% accuracy, suggesting the feasibility of using it to classify the tumor stroma ratio (TSR). Xu et al. [12] improved the activation features of the AlexNet model and proposed the characteristic of visualizing the neurons in the last hidden layer to classify and segment them. Trained by ImageNet, the framework successfully transferred the features extracted from the network into little histopathology images features for training and visualization and a test accuracy rate of 97.5\% was reported. Bejnordi et al. [13] proposed deep convolutional neural networks with some new geometric features, and trained the algorithm networks to classify stroma images, including stroma, fat tissues, other situ lesions and to predict the stroma regions. Bejnordi analyzed the stroma between surrounding invasive cancer and situ lesions and achieved a 96.2\% accuracy. Additionally, Kather [3] replaced the classification layer and the best accuracy rate was $98.7 \%$ with VGG19.

\subsection{Advantages and Limitations of Using Machine Learning Approaches}

Machine learning teaches computers to simulate and implement human learning behavior based on computational methods to learn knowledge from sample data. It is widely used in various applications such as image, content recommendation, computer vision, etc., in which it is difficult to develop conventional algorithms and solve the above problems to achieve the required tasks [14]. There are two main techniques: supervised learning (used to learn mapping between input and output) and unsupervised learning (involves using a model to extract relationships from data). The goals of machine learning are feature extraction, selection, prediction and recognition. The detailed processes are shown in Figure 1. This technology can automatically learn knowledge based on the data process in order to make accurate reaction, which generally can save a great deal of time.

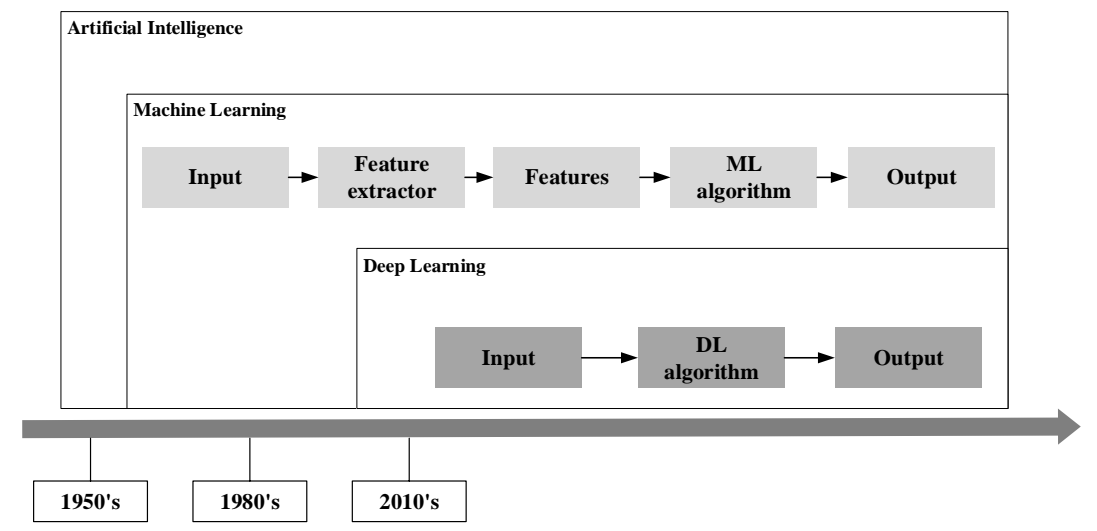

Figure 1. The trend of machine learning. 
The deep learning approach generally requires massive amount of data for training which means that the more data there is to train a model, the better it will perform. However, experts are needed for manual identification and labelling of histological images. It subjects to potential time-consumption and high expense issues. Even the underlying method automatically pays attention to discriminative information for better classification, prospective validation studies are still required to firmly establish routine biomarker for clinical use. In short, highly trained pathologists remain the decision-makers during the subjective evaluation for cancer diagnosis. The techniques developed by deep learning can assist the doctors for more accurate projection but not to replace the duty of physicians.

\subsection{How Deep Learning Works}

Deep Learning is another major subfield of machine learning. Hubel [15] used it to find corresponding relationships between neuron systems based on the cortex cells. Deep learning is inspired by biological nervous systems, and combine multiple nonlinear processing layers and hidden layers to learn features directly from data. Hinton [16,17] proposed that using multi-hidden layers to learn features is conducive to classification, as shown in Figure 2.

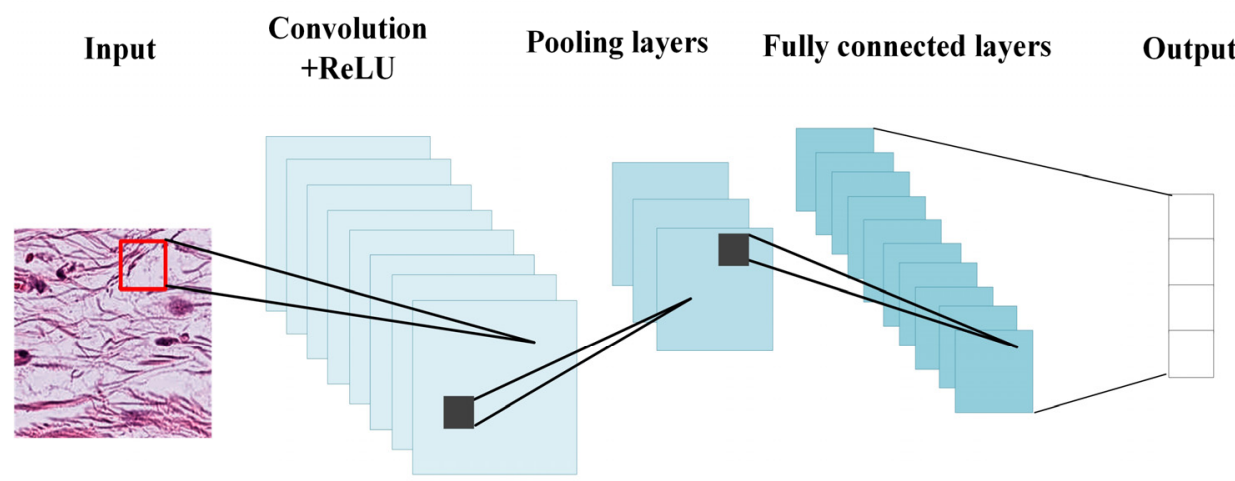

Feature Extraction

Classification

Figure 2. Network architecture of CNN.

The use of deep learning to learn features from multi-hidden layers of a large volume of data enhances the accuracy of predictions and a set of labels can be produced by using a GPU to train this model. Back propagation facilitates statistical regularity. Deep learning is based on the concept of learning from the first layer and automatically learning the features of many images from the combined layers. Each layer uses the output of the previous layer as input and learns to classify new images through to the next layer, and make a prediction.

Many different deep learning models have been developed for image recognition [18] over the past few years, such as histopathological image, facial recognition, and many advanced driver assistance technologies. CNN, which was proposed by Lecun [18,19], has a shared weights multi-neural layer network. The image is directly used as the input, which can reduce the complexity and parameters of the network. Besides, the structure of the network is invariant for image recognition. It is usually composed of two independent neurons, the first of which is an $S$ layer of the characteristic extraction as the number of input connections to the mode, and the neurons are of equal weight. The feature map has displacement invariance, being activated by the small sigmoid function. Another neuron, feature $C$ layer, is the feature extraction layer for anti-deformation. When part of the feature is extracted, its positional relationship with each input neuron connects with the previous layer.

A basic CNN consists of an input layer, output layer, and hidden layers, including ReLU, pooling layers, and fully connected layers: 
(1) Input layer: the input layer is the beginning of the artificial neural network, and brings the initial data, which comprises of a number of images, height, width, input channels, etc., into the system for further processing by subsequent layers of artificial neurons.

(2) Convolutional layers: This layer is used to extract the various features from the input images, such as corners and edges. In this layer, the operation of convolution is performed between the input image and a filter of an image size, which are used to operate the convolution of the input data and set the stride of pixels to scan the full image. Later, this feature map is fed into other layers to learn several other features of the input image.

(3) Rectified Linear Unit (ReLU): ReLU is the most common activation function in artificial neural networks. It is also known as transfer for better gradient propagation and efficient computation. $f(x)=\max (0, x)$. This function is defined as the positive part of its argument, where $x$ is the input to a neuron, the function used to determine the output of the neural network, and then maps the resulting values between 0 to 1 .

(4) Pooling layers: The pooling layers provide an approach to address this sensitivity to down sampling features by summarizing their presence in patches of the feature map. This capability of local translation invariance has the effect of making the resulting features focus on changes in the position of the featured images.

(5) Fully connected layers: This layer is the end of the network. It is often accompanied by the classifier to make the classification decision, and can be stacked.

\subsection{CNN Architecture and Models}

\subsubsection{CNN Architecture}

The structure of the CNN is designed for different purposes [20]. As seen in common neural networks, the neurons in a fully connected layer can be stacked and related to activations in the previous layer. This puts the filtered image on a higher level and puts it into a vote. With each additional layer, the network can learn more complex combinations of functions, which helps to make better decisions. These votes are expressed as the weight of the connection between each value or category. Therefore, their activation can be calculated by matrix multiplication and bias offset, and the main operations are performed by the backpropagation algorithm and the random gradient descent of the momentum algorithm, and fed back to the optimization method of weight update [20].

$\mathrm{CNN}$ is a cascaded filter, the first block of which is dedicated to detecting lower-level features (such as sharp points and surfaces folds), and the subsequent one is aggregated by the previous activation. From the perspective of deep learning, the main advantage of this architecture compared to traditional networks is that it can reduce the parameters of the image. $\mathrm{CNN}$ is composed of multiple connected kernels. Continuous layer learning features are gradually improved at the abstract level, and the input information can be represented hierarchically by combining low-level and high-level features. The objective of the fully connected layer is to take the results of the convolution to classify the images.

Currently, CNN [21] is widely used for image recognition by deep learning methods. Convolutional neural networks replace feature extraction, feature selection and classification. Different combinations of convolutional layers contain a series of fixed-size filters, which are used to manipulate the convolution of input data to generate so-called feature value maps (feature maps). During training in the context of convolutional neural networks, these filters can provide useful modules for image recognition, such as line detectors, regular edges and changes in image color. The ReLU layer usually follows the operation of the convolutional layer and provides a non-saturated activation function $f(x)=\max (0, x)$ for the output. According to Krizhevsky's research [22], these equations can be used to train fast-converging neural network convolutions, and can also solve the gradient problem, thereby accelerating the training. 


\subsubsection{Five Different CNN Models Networks}

In this paper, we used five common deep neural networks based on $\mathrm{CNN}$ models and proposed an improved classification model for systematic colorectal cancer (CRC) tissue.

\section{AlexNet}

AlexNet [22] is a widely-applied deep convolutional neural network, which can still achieve a competitive performance in classification compared to other kinds of networks. In the training stage of the AlexNet model, the input image is resized to $224 \times 224$ pixels and fed into the network. The architecture of AlexNet firstly adopts a convolutional layer to perform convolution and max pooling with local response normalization (LRN) using 96 different size $11 \times 11$ receptive filters. The max pooling operations are performed with $3 \times 3$ filters with a stride size of 2 . The same operations are performed in the second layer with $5 \times 5$ filters. The $3 \times 3$ filters are used in the third, fourth and fifth convolutional layers with 384, 384, and 296 feature maps respectively. The output of the two fully connected (FC) layers is used as an extracted feature vector with dropout followed by a softmax layer at the end.

\section{SqueezeNet}

SqueezeNet [23] is a small CNN architecture, which achieves AlexNet-level accuracy on ImageNet with 50x fewer parameters. Additionally, model compression techniques enabled us to compress SqueezeNet to less than $0.5 \mathrm{MB}(510 \times$ smaller than AlexNet). The SqueezeNet begins with a standalone convolution layer (conv1), followed by 8 Fire modules (fire2-9), ending with a final convolution layer (conv10). We gradually increased the number of filters per fire module from the beginning to the end of the network. SqueezeNet performed max-pooling with a stride of 2 after layers conv1, fire4, fire8, and conv10.

\section{VGGNet}

VGGNET [24] was the runner up of the 2014 ImageNet Large Scale Visual Recognition Challenge (ILSVRC). The main contribution of VGGNET is that it shows that the depth of a network is a critical component to achieve better recognition or classification accuracy in CNNs. The VGG architecture consists of two convolutional layers both of which use the ReLU activation function, which is followed by a single max pooling layer and several fully connected layers, which also use the ReLU activation function. The final layer of the VGGNet model is a Softmax layer for classification. In addition, the size of the convolution filter is changed to a $3 \times 3$ filter with a span of 2 in VGG-E.

\section{GoogLeNet}

The GoogLeNet [25] main architecture improves the computing resources inside the network model to incorporate inception layers with the objective and reducing complexity. It not only increases the depth of the architectural approach (adding $1 \times 1$ convolutional layers to the network) with a different kernel, but also the width of the network. This reduces the number of computation layers to capture sparse correlation patterns.

\section{ResNet}

The ResNet [26] has a residual learning framework with ultra-deep networks, and the residual functions can ease networks that did not lose out from a vanishing gradient problem. Unexpectedly, as the depth of the ResNet framework network increases, the accuracy is saturated, but adding more layers causes training errors.

\section{Research Method}

Deep learning has gained enormous popularity in scientific computing due to CNN, and its algorithms are widely used by industries to solve complex problems. In this study, we observed different network architectures [22-26] for comparison purposes. 


\subsection{Experimental Steps}

The diagram in Figure 3 illustrates the recognition process, which can be divided into three stages. The first stage is model training, the second is finding the superior architecture and parameters, and the third is model testing: 


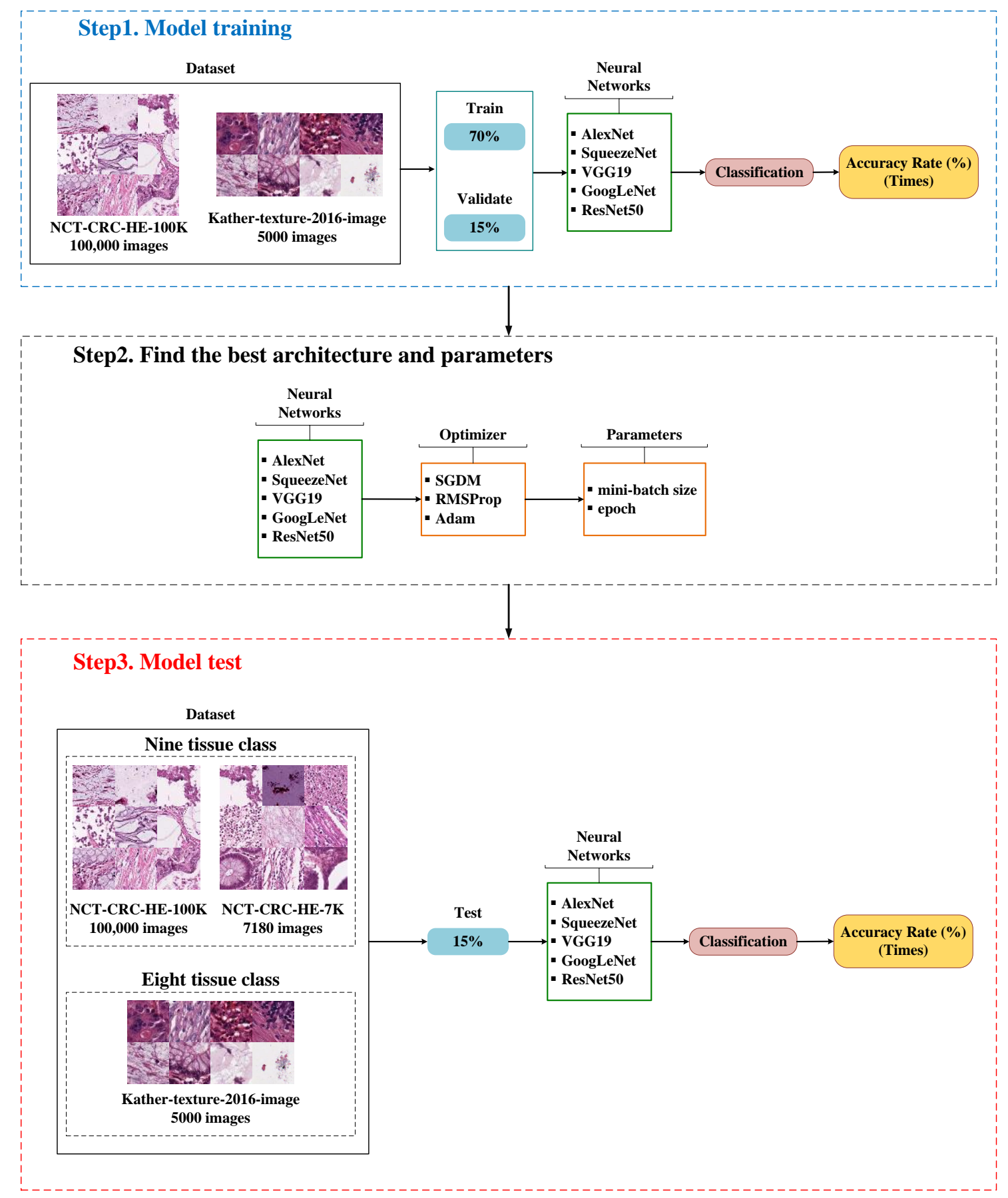

Figure 3. Deep learning structures to identify colorectal cancer tissue in this study.

Model Training

In this step, we used two different datasets. The first dataset we used was NCTCRC-HE-100K [3] image documents of histological images with a $150 \times 150$-pixel format, which included nine different tissue classes. We divided it into 70\% training dataset, 15\% validation dataset, and $15 \%$ test dataset. Since the number of each type in the original dataset was not the same, we used the number ratio of each type to take the corresponding number of training, validation and test for tissue classification in order to ensure the right proportion. Another dataset, the Kather-texture-2016-image [2] represents a collection of textures of eight tissue categories of human colorectal cancer in 5000 histological images of $150 \times 150$ pixels. Each image belongs to exactly one of the eight tissue categories, and the group sizes balanced (625 images per set). We divided it into 70\% training dataset, 15\% 
validation dataset, and $15 \%$ test dataset, as shown in Table 2. Secondly, we used 5 different CNN models for training: AlexNet [22], SqueezeNet [23], VGG19 [24], GoogLeNet [25], and Resnet50 [26].

Table 2. CRC dataset of histological images.

\begin{tabular}{|c|c|c|c|c|c|c|c|c|c|}
\hline \multirow{2}{*}{ Dataset } & \multirow{2}{*}{ Diagnosis } & \multicolumn{2}{|c|}{ Entire } & \multicolumn{2}{|c|}{ Training } & \multicolumn{2}{|c|}{ Validate } & \multicolumn{2}{|c|}{ Testing } \\
\hline & & \# WSI & $(\%)$ & \# WSI & $(\%)$ & \# WSI & $(\%)$ & \# WSI & $(\%)$ \\
\hline \multirow{9}{*}{ NCT-CRC-HE-100K [3] } & ADI & 10,407 & 10.41 & 7285 & 10.41 & 1561 & 10.41 & 1561 & 10.41 \\
\hline & BACK & 10,566 & 10.57 & 7396 & 10.57 & 1585 & 10.57 & 1585 & 10.57 \\
\hline & DEB & 11,512 & 11.51 & 8058 & 11.51 & 1727 & 11.51 & 1727 & 11.51 \\
\hline & LYM & 11,557 & 11.56 & 8090 & 11.56 & 1734 & 11.56 & 1734 & 11.56 \\
\hline & MUC & 8896 & 8.90 & 6227 & 8.90 & 1334 & 8.90 & 1334 & 8.90 \\
\hline & MUS & 13,536 & 13.54 & 9475 & 13.54 & 2030 & 13.54 & 2030 & 13.54 \\
\hline & NORM & 8763 & 8.76 & 6134 & 8.76 & 1314 & 8.76 & 1314 & 8.76 \\
\hline & STR & 10,446 & 10.45 & 7312 & 10.45 & 1567 & 10.45 & 1567 & 10.45 \\
\hline & TUM & 14,317 & 14.32 & 10,022 & 14.32 & 2148 & 14.32 & 2148 & 14.32 \\
\hline \multirow{9}{*}{ CRC-VAL-HE-7K [3] } & ADI & 1338 & 18.64 & 0 & 0 & 0 & 0 & 1338 & 18.64 \\
\hline & BACK & 847 & 11.80 & 0 & 0 & 0 & 0 & 847 & 11.80 \\
\hline & DEB & 339 & 4.72 & 0 & 0 & 0 & 0 & 339 & 4.72 \\
\hline & LYM & 634 & 8.83 & 0 & 0 & 0 & 0 & 634 & 8.83 \\
\hline & MUC & 1035 & 14.42 & 0 & 0 & 0 & 0 & 1035 & 14.42 \\
\hline & MUS & 592 & 8.25 & 0 & 0 & 0 & 0 & 592 & 8.25 \\
\hline & NORM & 741 & 10.32 & 0 & 0 & 0 & 0 & 741 & 10.32 \\
\hline & STR & 421 & 5.86 & 0 & 0 & 0 & 0 & 421 & 5.86 \\
\hline & TUM & 1233 & 17.17 & 0 & 0 & 0 & 0 & 1233 & 17.17 \\
\hline \multirow{8}{*}{ Kather-texture-2016-image [2] } & TUMOR & 625 & 78.125 & 468 & 12.48 & 93 & 12.48 & 93 & 12.48 \\
\hline & STROMA & 625 & 78.125 & 468 & 12.48 & 93 & 12.48 & 93 & 12.48 \\
\hline & COMPLEX & 625 & 78.125 & 468 & 12.48 & 93 & 12.48 & 93 & 12.48 \\
\hline & LYMPHO & 625 & 78.125 & 468 & 12.48 & 93 & 12.48 & 93 & 12.48 \\
\hline & DEBRIS & 625 & 78.125 & 468 & 12.48 & 93 & 12.48 & 93 & 12.48 \\
\hline & MUCOSA & 625 & 78.125 & 468 & 12.48 & 93 & 12.48 & 93 & 12.48 \\
\hline & ADIPOSE & 625 & 78.125 & 468 & 12.48 & 93 & 12.48 & 93 & 12.48 \\
\hline & EMPTY & 625 & 78.125 & 468 & 12.48 & 93 & 12.48 & 93 & 12.48 \\
\hline
\end{tabular}

Finding the Superior Architecture and Parameters

To gauge the performance of the network architectures of these CNN models, in the first research experiment we compared three training network method optimizers: stochastic gradient descent with momentum (SGDM), root mean square propagation (RMSProp) and adaptive moment estimation (Adam) with an NCT-CRC-HE-100K [3] dataset. As a result, the root-mean-square prop (RMSprop) method used the training options as input to the argument to select the training network to train, validate and test the CNN model to the highest accuracy, as shown in Table 3. In the second experiment, we trained the 5 different CNN models neural network with the root-mean-square prop (RMSprop) method. We further compared the replaced mini-batch size and epoch to test the models. 
Table 3. Comparison of network optimizers (CRC-VAL-HE-100K).

\begin{tabular}{|c|c|c|c|c|c|c|}
\hline \multicolumn{2}{|c|}{$\begin{array}{c}\text { Accuracy Rate (\%) } \\
\text { (Times) }\end{array}$} & \multicolumn{5}{|c|}{ Mini-Batch Size } \\
\hline Algorithms & Epoch & 8 & 16 & 32 & 64 & 128 \\
\hline \multirow{5}{*}{ sgdm } & 30 & $\begin{array}{c}95.08 \\
(668 \min 16 \mathrm{~s})\end{array}$ & $\begin{array}{c}95.21 \\
(709 \min 44 \mathrm{~s})\end{array}$ & $\begin{array}{c}95.36 \\
(761 \min 6 \mathrm{~s})\end{array}$ & $\begin{array}{c}95.11 \\
(821 \min 44 \mathrm{~s})\end{array}$ & $\begin{array}{c}95.17 \\
(889 \min 22 \mathrm{~s})\end{array}$ \\
\hline & 25 & $\begin{array}{c}95.09 \\
(659 \min 31 \mathrm{~s}) \\
\end{array}$ & $\begin{array}{c}95.19 \\
(701 \min 25 \mathrm{~s}) \\
\end{array}$ & $\begin{array}{c}95.35 \\
(748 \min 55 \mathrm{~s}) \\
\end{array}$ & $\begin{array}{c}95.17 \\
(812 \min 53 \mathrm{~s})\end{array}$ & $\begin{array}{c}95.16 \\
(874 \min 37 \mathrm{~s})\end{array}$ \\
\hline & 20 & $\begin{array}{c}95.02 \\
(647 \min 22 \mathrm{~s})\end{array}$ & $\begin{array}{c}95.24 \\
(691 \min 15 \mathrm{~s})\end{array}$ & $\begin{array}{c}95.37 \\
(741 \mathrm{~min} 32 \mathrm{~s})\end{array}$ & $\begin{array}{c}95.19 \\
(809 \min 47 \mathrm{~s})\end{array}$ & $\begin{array}{c}95.17 \\
(870 \min 49 \mathrm{~s})\end{array}$ \\
\hline & 15 & $\begin{array}{c}94.54 \\
(611 \min 15 \mathrm{~s})\end{array}$ & $\begin{array}{c}95.24 \\
(689 \min 46 \mathrm{~s})\end{array}$ & $\begin{array}{c}95.37 \\
(733 \min 27 \mathrm{~s})\end{array}$ & $\begin{array}{c}95.21 \\
(807 \min 32 \mathrm{~s})\end{array}$ & $\begin{array}{c}95.17 \\
(869 \min 18 \mathrm{~s})\end{array}$ \\
\hline & 10 & $\begin{array}{c}94.14 \\
(592 \min 46 \mathrm{~s})\end{array}$ & $\begin{array}{c}94.95 \\
(680 \min 21 \mathrm{~s})\end{array}$ & $\begin{array}{c}95.31 \\
(726 \min 41 \mathrm{~s}) \\
\end{array}$ & $\begin{array}{c}95.14 \\
(806 \min 58 \mathrm{~s})\end{array}$ & $\begin{array}{c}95.12 \\
(858 \min 45 \mathrm{~s})\end{array}$ \\
\hline \multirow{5}{*}{ rmsprop } & 30 & $\begin{array}{c}96.35 \\
(853 \min 24 \mathrm{~s})\end{array}$ & $\begin{array}{c}96.85 \\
(852 \min 17 \mathrm{~s})\end{array}$ & $\begin{array}{c}97.09 \\
(805 \min 53 \mathrm{~s})\end{array}$ & $\begin{array}{c}97.13 \\
(828 \min 44 \mathrm{~s})\end{array}$ & $\begin{array}{c}96.93 \\
(912 \min 17 \mathrm{~s})\end{array}$ \\
\hline & 25 & $\begin{array}{c}96.34 \\
(850 \min 18 \mathrm{~s})\end{array}$ & $\begin{array}{c}96.88 \\
(841 \mathrm{~min} 34 \mathrm{~s})\end{array}$ & $\begin{array}{c}97.22 \\
(892 \min 41 \mathrm{~s})\end{array}$ & $\begin{array}{c}97.14 \\
(897 \min 13 \mathrm{~s})\end{array}$ & $\begin{array}{c}96.98 \\
(901 \min 28 \mathrm{~s})\end{array}$ \\
\hline & 20 & $\begin{array}{c}96.32 \\
(847 \min 6 \mathrm{~s})\end{array}$ & $\begin{array}{c}96.88 \\
(838 \min 21 \mathrm{~s})\end{array}$ & $\begin{array}{c}97.23 \\
(867 \min 44 \mathrm{~s}) \\
\end{array}$ & $\begin{array}{c}97.14 \\
(893 \min 36 \mathrm{~s})\end{array}$ & $\begin{array}{c}97.01 \\
(900 \min 39 \mathrm{~s})\end{array}$ \\
\hline & 15 & $\begin{array}{c}96.32 \\
(842 \min 11 \mathrm{~s})\end{array}$ & $\begin{array}{c}96.85 \\
(831 \min 19 \mathrm{~s})\end{array}$ & $\begin{array}{c}97.22 \\
(871 \min 12 \mathrm{~s})\end{array}$ & $\begin{array}{c}97.14 \\
(890 \min 11 \mathrm{~s})\end{array}$ & $\begin{array}{c}97.03 \\
(899 \min 47 \mathrm{~s})\end{array}$ \\
\hline & 10 & $\begin{array}{c}96.32 \\
(838 \min 37 \mathrm{~s})\end{array}$ & $\begin{array}{c}96.69 \\
(820 \min 47 \mathrm{~s})\end{array}$ & $\begin{array}{c}97.08 \\
(869 \min 44 \mathrm{~s})\end{array}$ & $\begin{array}{c}97.01 \\
(884 \min 19 \mathrm{~s})\end{array}$ & $\begin{array}{c}97.03 \\
(898 \min 7 \mathrm{~s})\end{array}$ \\
\hline \multirow{5}{*}{ adam } & 30 & $\begin{array}{c}95.28 \\
(814 \min 8 \mathrm{~s})\end{array}$ & $\begin{array}{c}95.59 \\
(824 \min 33 \mathrm{~s})\end{array}$ & $\begin{array}{c}96.36 \\
(807 \min 22 \mathrm{~s})\end{array}$ & $\begin{array}{c}95.38 \\
(830 \min 45 \mathrm{~s})\end{array}$ & $\begin{array}{c}94.20 \\
(824 \min 37 \mathrm{~s})\end{array}$ \\
\hline & 25 & $\begin{array}{c}95.29 \\
(810 \min 39 \mathrm{~s})\end{array}$ & $\begin{array}{c}95.41 \\
(822 \min 14 \mathrm{~s})\end{array}$ & $\begin{array}{c}96.36 \\
(806 \min 35 \mathrm{~s})\end{array}$ & $\begin{array}{c}95.37 \\
(818 \min 50 \mathrm{~s})\end{array}$ & $\begin{array}{c}94.17 \\
(820 \min 49 \mathrm{~s})\end{array}$ \\
\hline & 20 & $\begin{array}{c}95.28 \\
(807 \min 52 \mathrm{~s})\end{array}$ & $\begin{array}{c}95.38 \\
(812 \min 26 \mathrm{~s})\end{array}$ & $\begin{array}{c}96.37 \\
(802 \min 42 \mathrm{~s})\end{array}$ & $\begin{array}{c}95.37 \\
(814 \min 59 \mathrm{~s}) \\
\end{array}$ & $\begin{array}{c}94.19 \\
(820 \min 17 \mathrm{~s})\end{array}$ \\
\hline & 15 & $\begin{array}{c}95.27 \\
(796 \min 6 \mathrm{~s})\end{array}$ & $\begin{array}{c}95.38 \\
(800 \min 32 \mathrm{~s})\end{array}$ & $\begin{array}{c}96.37 \\
(803 \min 15 \mathrm{~s})\end{array}$ & $\begin{array}{c}95.39 \\
(809 \min 38 \mathrm{~s})\end{array}$ & $\begin{array}{c}94.21 \\
(815 \min 29 \mathrm{~s})\end{array}$ \\
\hline & 10 & $\begin{array}{c}95.27 \\
(790 \min 28 \mathrm{~s})\end{array}$ & $\begin{array}{c}95.38 \\
(798 \min 38 \mathrm{~s})\end{array}$ & $\begin{array}{c}95.36 \\
(808 \min 14 \mathrm{~s})\end{array}$ & $\begin{array}{c}95.38 \\
(809 \min 9 \mathrm{~s})\end{array}$ & $\begin{array}{c}93.21 \\
(811 \min 8 \mathrm{~s})\end{array}$ \\
\hline
\end{tabular}

Bold symbols represent the maximum values of each column in the tables.

Model Testing

The last stage of our architectural parameters involved classifying the histological images through each CNN model's neural network architecture by training the model to identify the types from different tissue classes. After neural network training all the 100,000 image patches (which were derived from 86 whole-slide images) in the first dataset (NCT-CRC-HE-100K), we used the dataset for testing purposes. Besides, we assessed the accuracy of the tissue classification and the convolutional neural network using an independent external dataset (CRC-VAL-HE-7K), which contained 7180 image patches derived from 25 hematoxylin and eosin (H\&E) slides of human CRC tissue. Additionally, we used $70 \%$ of the dataset (Kather-texture-2016-image) consisting of 5000 images in eight classes of colorectal cancer tissue for training, $15 \%$ for validation and the other $15 \%$ for testing. We created a confusion matrix chart of the experimental results and showed the precision of each class using column and row summaries. The percentage of correctly and incorrectly classified observations for the true class were shown in the normalized row, 
while the percentage of correctly and incorrectly classified observations for the predicted class were shown in the normalized column.

\subsection{Data Availability}

\subsubsection{Images of Nine Tissue Classes}

In this experiment, we used the open histological dataset of nine tissue classes from NCT-CRC-HE-100K for model training. These images were generated by Kather et al. [3], and have 86 hematoxylin and eosin stain (H\&E) slides of tissue. The labels of the histological images of the available data were taken from the NCT-UMM website. The example images of the nine tissue classes are listed in Figure 4. The dimensions of all the images were $224 \times 224$ pixels $(112 \times 112 \mu \mathrm{m})$, and they were presented to the model network sequentially for training, validation and testing. After training and testing our network framework with the "NCT-CRC-HE-100K", we also assessed the accuracy of the tissue classification with an external validation set, data description "CRC-VAL-HE-7K", which contained 7180 image patches for testing purposes only. The nine classes are categorized as following (Figure 4):

(a) ADI: adipose tissue is mainly composed of adipocytes.

(b) BACK: histological image background.

(c) DEB: debris is widely used in histopathology and diagnoses.

(d) LYM: lymphocytes are the main type of cells found in the lymphatic system.

(e) MUC: mucus is produced by many tissues in the body, and acts as a protective force.

(f) MUS: smooth muscle.

(g) NORM TISSUE: tissues of colon mucosa.

(h) STR: stroma tissues associated with cancer.

(i) TUM: epithelium tissues of adenocarcinoma.

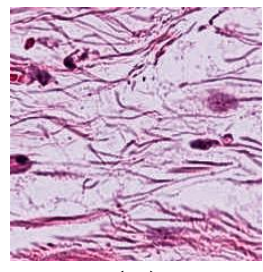

(a)

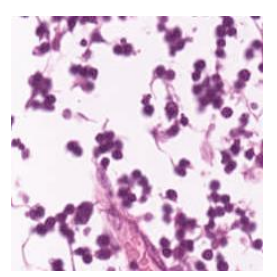

(d)

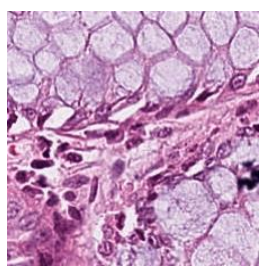

(g)

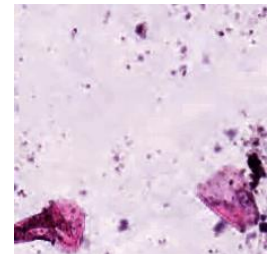

(b)

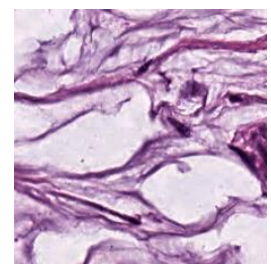

(e)

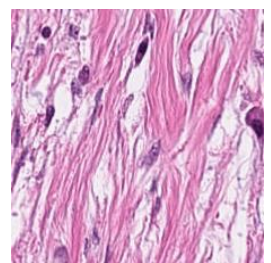

(h)

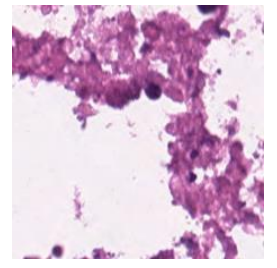

(c)

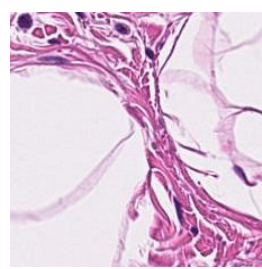

(f)

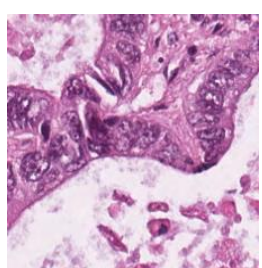

(i)

Figure 4. Example images of the nine tissue classes represented in the NCT-CRC-HE-100K dataset. (a-i) defined in Section 3.2.1. 


\subsubsection{Images of Eight Tissue Classes}

We used the open dataset Kather-texture-2016-image to verify the accuracy of our optimized deep neural network architecture in distinguishing other tissue classes. This dataset was collected from the Institute of Histological Images of Pathology of Human Colorectal Cancer taken from the pathology archive by Kather, et al. [2]. The dataset consisted of 5000 non-duplicated histological images of human colorectal cancer (CRC) using hematoxylin and eosin (H\&E) and healthy normal tissue images. This dataset created images with $150 \times 150$ pixels $(74 \times 74 \mu \mathrm{m})$ each for every RGB color, and contained eight different tissue texture features and original tissue images with a size of 5000 pixels (e.g., Figure 5).

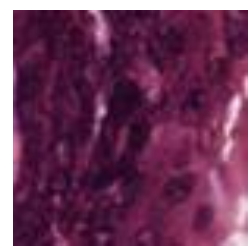

(a)

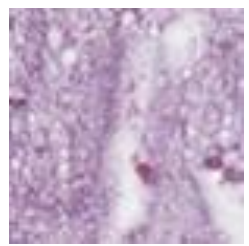

(e)

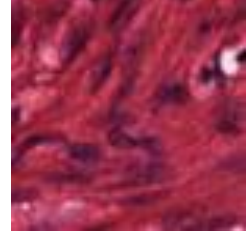

(b)

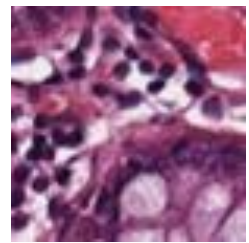

(f)

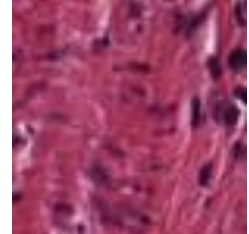

(c)

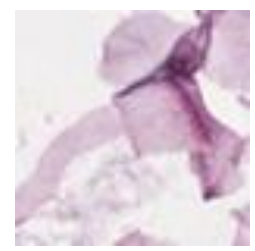

(g)

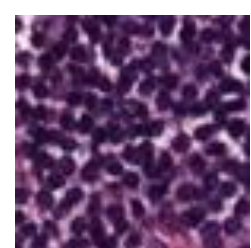

(d)

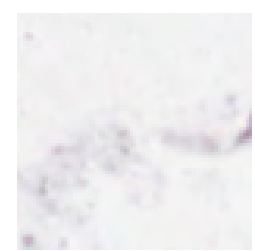

(h)

Figure 5. Example images of the eight tissue classes represented in the Kather-texture-2016-image5000 dataset. (a-h) defined in Section 3.2.2.

The eight classes are categorized as following (Figure 5):

(a) TUMOR: a tumor is an abnormal new growth of cells.

(b) STROMA: stroma is the part of a tissue or organ with a structural or connective role.

(c) COMPLEX: complex stroma contain a single or a few tissue cells.

(d) LYMPHO: lymphoma is a group of blood malignancies that develop from lymphocytes.

(e) DEBRIS: debris or H\&E stain is one of the principal tissue stains used in histology.

(f) MUCOSA: mucus is produced by many tissues in the body, and acts as a protective force.

(g) ADIPOSE: adipose tissue is mainly composed of adipocytes.

(h) EMPTY: histological image background.

\subsection{Software and Tools Platform}

In this study, we used the MATLAB of R2020a based on deep neural network architecture to train and test on two Intel workstation computers with high-level NVIDIA QUADRO GeForce GTX 1070 GPUs, and OS Windows 10 64-bit Core i7 i7-7700 with $3.60 \mathrm{GHz}$ Processor (4 Core).

\section{Experiments and Discussion}

A series of experiments on different convolutional neural networks (CNNs) models were conducted in this study, including AlexNet, SqueezeNet, VGGNet, GoogLeNet and ResNet50. In Experiment I, we compared the accuracy rate of three training network optimizer methods: the stochastic gradient descent with momentum (SGDM), the root mean square propagation (RMSProp), which utilizes the magnitude of recent gradients to normalize the gradients, and the adaptive moment estimation (Adam), which is an 
optimization algorithm that can be used for a classical stochastic gradient descent. In addition, parts of the parameters in the network layers were modified, such as the minibatch size and different epoch. Next, we used our approach to identify the accuracy rate of the colorectal cancer tissue types from the histological images in different open datasets, and the results are presented in the next section.

\subsection{Experiment I: Comparing the Accuracy Rate of Network Optimizers \\ 4.1.1. Approach \\ Load and Explore Image Data}

Firstly, we loaded three different open datasets of histological images. The first dataset consisted of 100,000 histological images (NCT-CRC-HE-100K) of colorectal cancer, including nine classes of tissues. The second dataset was (CRC-VAL-HE-7K), which contained 7180 image patches. The last dataset consisted of 5000 histological images of colorectal cancer, including eight different types of tissue. The image store automatically labeled the images based on folder names and efficiently read batches of images while training a convolutional neural network.

\section{Randomly Split the Dataset}

Next, we split the image dataset into three data stores: $70 \%$ into training data and $15 \%$ each into testing and validation, so that none of them overlapped with the others.

Define the Convolutional Neural Network Architecture

At this stage, we used 5 different CNN models for the training dataset: AlexNet [22], SqueezeNet [23], VGG19 [24], GoogLeNet [25], and Resnet50 [26]. The architecture included different convolutional layers, rectified linear units layer (ReLU layer), max-pooling layer, and fully connected layers.

Specify a Set of Options for Training

The network was trained using stochastic gradient descent with three training method optimizers after defining the network structure: stochastic gradient descent with momentum (SGDM), root mean square propagation (RMSProp), adaptive moment estimation (Adam) with an initial learning rate of 0.01 and four training periods on the entire dataset.

Train the Network

Train the network of histological images, and monitor the accuracy rate.

Predict Classification Accuracy

Predict the test data with three open datasets to calculate the final accuracy rate and execution time.

\subsubsection{Experimental Results}

Since deep learning technique will be adopted in this study, the performance of a CNN model depends on many factors in general. For example, the weight initialization, batch sizes, epochs, learning rates, activation function, optimizer, loss function, network topology, etc. The optimizer selection study of [27] for brain tumor segmentation in magnetic resonance images (MRI) suggests that a good optimizer could be a critical issue for the proposed approach. The authors of [27] listed 10 different state-of-the-art optimizer including: adaptive gradient (Adagrad), adaptive delta (AdaDelta), stochastic gradient descent (SGD), adaptive momentum (Adam), cyclic learning rate (CLR), adaptive max pooling (Adamax), root mean square propagation (RMS Prop), Nesterov adaptive momentum (Nadam), and Nesterov accelerated gradient (NAG) for CNN. The Adam optimizer achieved the best accuracy in study of [27] for MRI. Comprehensive analyses have been performed during this study for those optimizers. Based on the performance of final results, only SGDM, 
RMSProp, Adam are listed since their performance are overall better than other optimizers for different network models.

Firstly, the open dataset description (Kather-texture-2016-image), which included 5000 images in eight tissue classes are trained and the results of the experiment are shown in Table 4, and the plot of the confusion matrix in Figure 6.

Table 4. Experiment I: the best results of eight tissue classes (Kather-texture-2016-image).

\begin{tabular}{|c|c|c|c|}
\hline $\begin{array}{c}\text { Accuracy Rate (\%) } \\
\text { (Times) }\end{array}$ & & Algorithms & \\
\hline Model & Sgdm & Rmsprop & Adam \\
\hline ResNet18 & $\begin{array}{c}91.89 \\
(17 \min 22 \mathrm{~s})\end{array}$ & $\begin{array}{c}92.41 \\
(21 \mathrm{~min} 15 \mathrm{~s})\end{array}$ & $\begin{array}{c}92.33 \\
(25 \min 51 \mathrm{~s})\end{array}$ \\
\hline ResNet50 & $\begin{array}{c}93.27 \\
(19 \min 12 \mathrm{~s})\end{array}$ & $\begin{array}{c}94.18 \\
(28 \operatorname{min~} 22 \mathrm{~s})\end{array}$ & $\begin{array}{c}94.02 \\
(33 \min 56 \mathrm{~s})\end{array}$ \\
\hline ResNet101 & $\begin{array}{c}92.98 \\
(21 \min 19 \mathrm{~s})\end{array}$ & $\begin{array}{c}94.11 \\
(32 \min 33 \mathrm{~s})\end{array}$ & $\begin{array}{c}93.99 \\
(43 \min 43 \mathrm{~s})\end{array}$ \\
\hline GoogLeNet & $\begin{array}{c}91.89 \\
(8 \min 25 s)\end{array}$ & $\begin{array}{c}92.17 \\
(11 \mathrm{~min} 11 \mathrm{~s})\end{array}$ & $\begin{array}{c}92.13 \\
(15 \min 29 \mathrm{~s})\end{array}$ \\
\hline VGG19 & $\begin{array}{c}88.91 \\
(11 \min 27 \mathrm{~s})\end{array}$ & $\begin{array}{c}90.85 \\
(15 \min 3 \mathrm{~s})\end{array}$ & $\begin{array}{c}89.94 \\
(17 \min 54 \mathrm{~s})\end{array}$ \\
\hline SqueezeNet & $\begin{array}{c}85.22 \\
(14 \min 57 \mathrm{~s})\end{array}$ & $\begin{array}{c}86.31 \\
(16 \mathrm{~min} 01 \mathrm{~s})\end{array}$ & $\begin{array}{c}86.11 \\
(19 \min 29 \mathrm{~s})\end{array}$ \\
\hline AlexNet & $\begin{array}{c}87.93 \\
(17 \min 27 \mathrm{~s})\end{array}$ & $\begin{array}{c}88.81 \\
(19 \min 36 \mathrm{~s})\end{array}$ & $\begin{array}{c}88.24 \\
(21 \min 17 \mathrm{~s})\end{array}$ \\
\hline
\end{tabular}

Bold symbols represent the maximum values of each row in the tables.

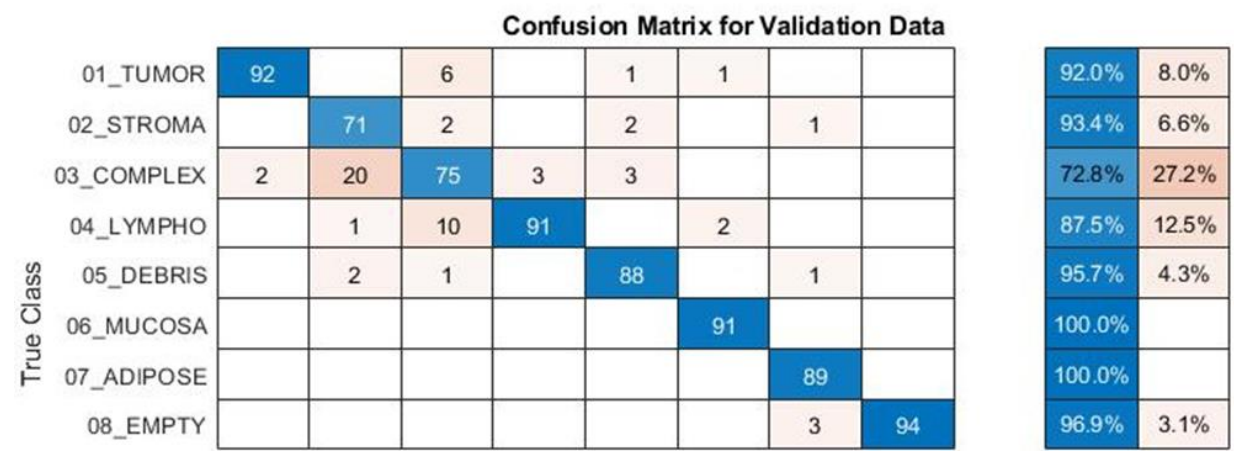

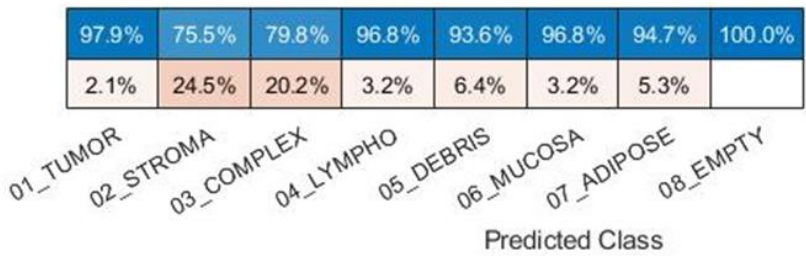

Figure 6. Experiment I: the accuracy of eight classes (Kather-texture-2016-image-5000).

Secondly, we used the data description (NCT-CRC-HE-100K) image documents with a $224 \times 224$-pixel format to classify the histological images, which included 100,000 images in nine different tissue classes, and display the precision for each class by using column and row summaries to plot the confusion matrix, as shown in Figure 7. In addition, we tested the classification performance with another independent set of 7000 images from different patients (CRC-VAL-HE-7K), and plotted the confusion matrix, as shown in Figure 8. The detailed results are shown in Table 5. 
Among the selected optimizer, unlike Adam achieved the highest accuracy for brain tumor segmentation in magnetic resonance images, root-mean-square prop (RMSprop) network optimizer consistently have the highest accuracy rates for colorectal cancer tissues shown in Tables 4 and 5. Therefore, RMSprop optimizer will be adopted in the following experiments.

\begin{tabular}{|c|c|c|c|c|c|c|c|c|c|c|c|}
\hline \multicolumn{10}{|c|}{ Confusion Matrix for Validation Data } & \multirow{3}{*}{$\frac{99.4 \%}{100.0 \%}$} & \multirow[b]{2}{*}{$0.6 \%$} \\
\hline$A D I$ & 1560 & & & & 2 & 2 & & 6 & & & \\
\hline BACK & & 1585 & & & & & & & & & \\
\hline DEB & & & 1708 & 5 & 2 & & & 3 & 8 & $99.0 \%$ & $1.0 \%$ \\
\hline LYM & & & & 1727 & & & & & 1 & $99.9 \%$ & $0.1 \%$ \\
\hline MUC & 1 & & & & 1325 & & 7 & 3 & 4 & $98.9 \%$ & $1.1 \%$ \\
\hline MUS & & & 2 & & & 2016 & & 11 & & $99.4 \%$ & $0.6 \%$ \\
\hline NORM & & & & 2 & & 1 & 1299 & & 3 & $99.5 \%$ & $0.5 \%$ \\
\hline STR & & & 17 & & 5 & 11 & 2 & 1543 & 12 & $97.0 \%$ & $3.0 \%$ \\
\hline TUM & & & & & & & 6 & 1 & 2120 & $99.7 \%$ & $0.3 \%$ \\
\hline
\end{tabular}

\begin{tabular}{|c|c|c|c|c|c|c|c|c|}
\hline $99.9 \%$ & $100.0 \%$ & $98.9 \%$ & $99.6 \%$ & $99.3 \%$ & $99.3 \%$ & $98.9 \%$ & $98.5 \%$ & $98.7 \%$ \\
\hline $0.1 \%$ & & $1.1 \%$ & $0.4 \%$ & $0.7 \%$ & $0.7 \%$ & $1.1 \%$ & $1.5 \%$ & $1.3 \%$ \\
\hline ADI & BACK & DEB & LYM & MUC & MUS NORM STR & TUM \\
& & \multicolumn{7}{c|}{ Predicted Class }
\end{tabular}

Figure 7. Experiment I: the accuracy of nine classes (CRC-VAL-HE-100K).

\begin{tabular}{|c|c|c|c|c|c|c|c|c|c|c|c|}
\hline \multicolumn{10}{|c|}{ Confusion Matrix for Validation Data } & \\
\hline ADI & 201 & & & & & & & & & & \\
\hline BACK & & 127 & & & & & & & & & \\
\hline DEB & & & 49 & & & & & & & $100.0 \%$ & \\
\hline LYM & & & & 94 & & & & 1 & & $98.9 \%$ & $1.1 \%$ \\
\hline MUC & & & & & 155 & & 1 & & & $99.4 \%$ & $0.6 \%$ \\
\hline$\frac{\pi}{0} \frac{\pi}{0}$ MUS & & & 2 & & & 89 & & 4 & & $93.7 \%$ & $6.3 \%$ \\
\hline NORM & & & & 1 & & & 109 & & & $99.1 \%$ & $0.9 \%$ \\
\hline STR & & & & & & & & 58 & & $100.0 \%$ & \\
\hline TUM & & & & & & & 1 & & 185 & $99.5 \%$ & $0.5 \%$ \\
\hline
\end{tabular}

\begin{tabular}{|l|l|l|l|l|l|l|l|l|}
\hline $100.0 \%$ & $100.0 \%$ & $96.1 \%$ & $98.9 \%$ & $100.0 \%$ & $100.0 \%$ & $98.2 \%$ & $92.1 \%$ & $100.0 \%$ \\
\hline
\end{tabular}

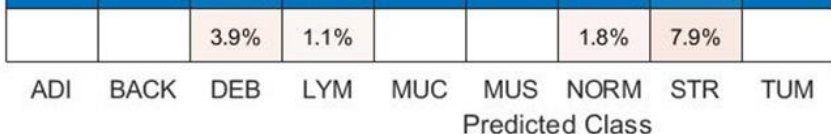

Figure 8. Experiment I: the accuracy of nine classes (CRC-VAL-HE-7K). 
Table 5. Experiment I: the best results of nine tissue classes (CRC-VAL-HE-7K).

\begin{tabular}{cccc}
\hline $\begin{array}{c}\text { Accuracy Rate (\%) } \\
\text { (Times) }\end{array}$ & Sgdm & Algorithms & \\
\hline Model & 94.81 & Rmsprop & Adam \\
\hline ResNet18 & $(6 \mathrm{~s})$ & $\mathbf{9 6 . 3 3}$ & 95.93 \\
& 96.94 & $\mathbf{( 1 1 ~ s )}$ & $(11 \mathrm{~s})$ \\
\hline \multirow{2}{*}{ ResNet50 } & $(11 \mathrm{~s})$ & $\mathbf{9 7 . 2 2}$ & 96.37 \\
& 95.35 & $\mathbf{( 1 0 ~ s )}$ & $(14 \mathrm{~s})$ \\
\hline \multirow{2}{*}{ ResNet101 } & $(14 \mathrm{~s})$ & $\mathbf{9 7 . 1 4}$ & 96.82 \\
& 95.89 & $\mathbf{( 1 3 ~ s )}$ & $(17 \mathrm{~s})$ \\
\hline \multirow{2}{*}{ GoogLeNet } & $(5 \mathrm{~s})$ & $\mathbf{9 7 . 0 5}$ & 96.18 \\
& 96.12 & $\mathbf{( 6 ~ s )}$ & 97.01 \\
\hline \multirow{2}{*}{ VGG19 } & $(3 \mathrm{~s})$ & $\mathbf{9 7 . 0 8}$ & $(7 \mathrm{~s})$ \\
\hline \multirow{2}{*}{ AlexNet } & 95.77 & $\mathbf{( 3 ~ s )}$ & 96.05 \\
& $(4 \mathrm{~s})$ & $\mathbf{9 6 . 0 4}$ & $(13 \mathrm{~s})$ \\
\hline
\end{tabular}

Bold symbols represent the maximum values of each row in the table.

\subsection{Experiment II: Our Trained Deep Learning Approaches}

\subsubsection{Approach}

Using the same split dataset method as shown in Experiment 1, we trained the neural network of five different $\mathrm{CNN}$ models with the most accurate optimizer: root-mean-square prop (RMSprop) and compared it with the replaced mini-batch size and epoch. Parts of the network layer were extracted to a new mode in the model revision process, which was used to extract the image features to modify the parameter. We improved this stage based on five cycles of the minimum batch size per training. We considered different models of convolutional neural networks (CNNs), such as AlexNet, SqueezeNet, VGGNet, GoogLeNet and ResNet for the classification of the pathological images.

The architectural design of the convolutional neural network (CNN) ResNet50 can be seen in Table 6. (ONV + POOL)_maxrepresents the convolutional layer, followed by the use of the maximum generalized pooling layer, and (CONV + POOL)_avg is a pooling layer that follows the generalization of the average.

Table 6. Summary of parameters for ResNet50.

\begin{tabular}{|c|c|c|c|c|}
\hline & Name & Type & Activations & $\begin{array}{c}\text { Learnable } \\
\text { Parameters }\end{array}$ \\
\hline 1 & $\begin{array}{c}\text { data } \\
224 \times 224 \times 3\end{array}$ & Image Input & $224 \times 224 \times 3$ & - \\
\hline 2 & Conv1-7 × 7_s2 & Convolution & $112 \times 112 \times 64$ & $\begin{array}{c}\text { Weights } 7 \times 7 \times 3 \\
\times 64 \\
\text { Bias } 1 \times 1 \times 64\end{array}$ \\
\hline 3 & Conv1-relu_7 $\times 7$ & ReLU & $112 \times 112 \times 64$ & - \\
\hline 4 & Pool-3 × 3_s2 & Max Pooling & $56 \times 56 \times 64$ & - \\
\hline 5 & Pool1-norm1 & $\begin{array}{l}\text { Cross Channel } \\
\text { Normalization }\end{array}$ & $56 \times 56 \times 64$ & - \\
\hline 6 & Conv2-3 $\times$ 3_reduce & Convolution & $56 \times 56 \times 64$ & $\begin{array}{c}\text { Weights } 1 \times 1 \times 3 \\
\times 64 \\
\text { Bias } 1 \times 1 \times 64\end{array}$ \\
\hline
\end{tabular}


Table 6. Cont.

\begin{tabular}{|c|c|c|c|c|}
\hline & Name & Type & Activations & $\begin{array}{c}\text { Learnable } \\
\text { Parameters }\end{array}$ \\
\hline 7 & $\begin{array}{c}\text { Conv2-relu_3 } \times \\
\text { 3_reduce }\end{array}$ & $\operatorname{ReLU}$ & $56 \times 56 \times 64$ & - \\
\hline 8 & Conv2-3 $\times 3$ & Convolution & $56 \times 56 \times 192$ & $\begin{array}{c}\text { Weights } 3 \times 3 \times 64 \\
\times 192 \\
\text { Bias } 1 \times 1 \times 192\end{array}$ \\
\hline 9 & Conv2-relu_3 × 3 & $\operatorname{ReLU}$ & $56 \times 56 \times 192$ & - \\
\hline 10 & Conv2-norm2 & $\begin{array}{l}\text { Cross Channel } \\
\text { Normalization }\end{array}$ & $56 \times 56 \times 192$ & - \\
\hline 11 & Pool2-3 × 3_s2 & Max Pooling & $28 \times 28 \times 192$ & - \\
\hline 12 & Inception_3a-1 $\times 1$ & Convolution & $28 \times 28 \times 64$ & $\begin{array}{c}\text { Weights } 1 \times 1 \times \\
192 \times 164 \\
\text { Bias } 1 \times 1 \times 64\end{array}$ \\
\hline$\vdots$ & $\vdots$ & $\vdots$ & $\vdots$ & $\vdots$ \\
\hline 144 & Output & $\begin{array}{c}\text { Classification } \\
\text { Output }\end{array}$ & - & - \\
\hline
\end{tabular}

\subsubsection{Experimental Results}

In the second part, we compared five different CNN networks with the replaced mini-batch size and epoch from the network to form a new model. We began by using the data "NCT-CRC-HE-100K" documents of histological images for the model training with a $224 \times$ 224-pixel format for classification, including 100,000 images of nine different tissue classes, and displaying the precision of each class by using column and row summaries to plot the confusion matrix, as shown in Figure 9. The detailed results are shown in Table 7. Secondly, we tested the classification performance in an independent dataset of 7180 images from "CRC-VAL-HE-7K", and plotted the confusion matrix as shown in Figure 10. The detailed results are shown in Table 8. In addition, we used the open dataset description "Kather-texture-2016-image", which included 5000 images in eight tissue classes. The experimental results are shown in Table 9, and the plot of the confusion matrix is shown in Figure 11.
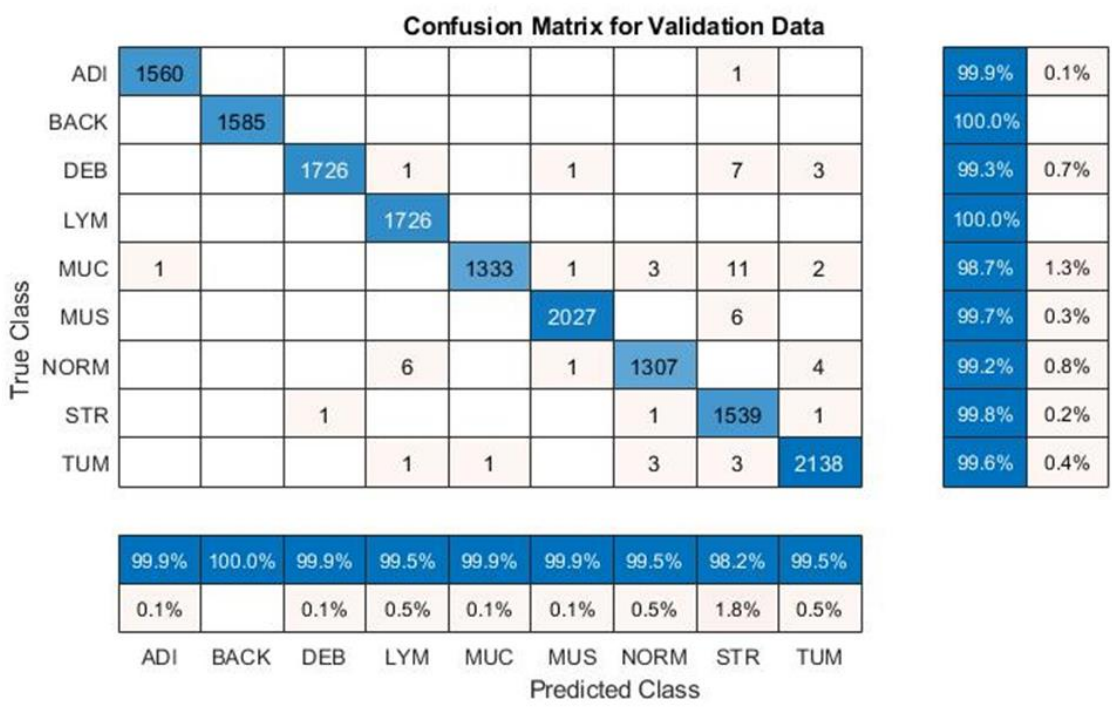

Figure 9. Experiment II: accuracy of nine classes (CRC-VAL-HE-100K). 
Table 7. Experiment II: the best results of nine tissue classes (CRC-VAL-HE-100K).

\begin{tabular}{|c|c|c|c|c|c|c|}
\hline \multicolumn{2}{|c|}{ Accuracy Rate (\%) (Times) } & \multicolumn{5}{|c|}{ Mini-Batch Size } \\
\hline Model & Epoch & 8 & 16 & 32 & 64 & 128 \\
\hline \multirow{5}{*}{ ResNet18 } & 30 & $98.12(689 \min 43 \mathrm{~s})$ & $98.27(707 \min 39 \mathrm{~s})$ & $98.60(709 \min 45 \mathrm{~s})$ & $98.65(711 \min 45 \mathrm{~s})$ & $98.48(720 \mathrm{~min} 08 \mathrm{~s})$ \\
\hline & 25 & $98.09(671 \min 22 \mathrm{~s})$ & $98.27(692 \min 44 \mathrm{~s})$ & $98.61(694 \min 17 \mathrm{~s})$ & $98.64(698 \min 55 \mathrm{~s})$ & $98.47(799 \min 49 \mathrm{~s})$ \\
\hline & 20 & $98.09(665 \min 19 \mathrm{~s})$ & $98.24(680 \min 18 \mathrm{~s})$ & $98.61(681 \min 22 \mathrm{~s})$ & $98.66(690 \min 29 \mathrm{~s})$ & $98.49(796 \min 05 \mathrm{~s})$ \\
\hline & 15 & $98.02(639 \min 28 \mathrm{~s})$ & $98.07(669 \min 27 \mathrm{~s})$ & $98.61(672 \min 18 \mathrm{~s})$ & $98.66(684 \min 05 \mathrm{~s})$ & $98.48(789 \min 34 \mathrm{~s})$ \\
\hline & 10 & $98.01(622 \min 17 \mathrm{~s})$ & $98.01(643 \min 19 \mathrm{~s})$ & $98.02(666 \min 16 \mathrm{~s})$ & $98.44(771 \mathrm{~min} 33 \mathrm{~s})$ & $98.48(770 \mathrm{~min} 31 \mathrm{~s})$ \\
\hline \multirow{5}{*}{ ResNet50 } & 30 & $98.35(757 \mathrm{~min} 08 \mathrm{~s})$ & $99.02(758 \min 31 \mathrm{~s})$ & $99.67(769 \mathrm{~min} 04 \mathrm{~s})$ & $99.46(790 \min 22 \mathrm{~s})$ & $99.41(792 \min 12 \mathrm{~s})$ \\
\hline & 25 & $98.29(739 \min 33 \mathrm{~s})$ & $99.02(756 \min 05 \mathrm{~s})$ & $99.68(762 \min 18 \mathrm{~s})$ & $99.46(788 \mathrm{~min} 09 \mathrm{~s})$ & $99.45(789 \min 55 \mathrm{~s})$ \\
\hline & 20 & $98.31(733 \min 29 \mathrm{~s})$ & $98.89(749 \min 22 \mathrm{~s})$ & $99.69(756 \mathrm{~min} 03 \mathrm{~s})$ & $99.46(781 \mathrm{~min} 26 \mathrm{~s})$ & $99.44(782 \min 17 \mathrm{~s})$ \\
\hline & 15 & $98.21(719 \min 16 \mathrm{~s})$ & $98.72(736 \min 05 \mathrm{~s})$ & 99.68 (731 min $07 \mathrm{~s})$ & $99.46(768 \min 17 \mathrm{~s})$ & $99.45(769 \min 38 \mathrm{~s})$ \\
\hline & 10 & $98.21(709 \min 15 \mathrm{~s})$ & $98.77(728 \min 17 \mathrm{~s})$ & $99.68(729 \min 19 \mathrm{~s})$ & $99.45(742 \min 11 \mathrm{~s})$ & $99.45(761 \mathrm{~min} 21 \mathrm{~s})$ \\
\hline \multirow{5}{*}{ ResNet101 } & 30 & $98.89(797 \min 21 \mathrm{~s})$ & $99.03(792 \min 27 \mathrm{~s})$ & $99.30(798 \min 41 \mathrm{~s})$ & $98.79(809 \min 39 \mathrm{~s})$ & $98.50(829 \min 10 \mathrm{~s})$ \\
\hline & 25 & $98.81(789 \min 17 \mathrm{~s})$ & $99.03(790 \mathrm{~min} 07 \mathrm{~s})$ & $99.31(793 \min 28 \mathrm{~s})$ & $98.77(795 \min 48 \mathrm{~s})$ & $98.54(827 \mathrm{~min} 01 \mathrm{~s})$ \\
\hline & 20 & $98.79(780 \min 54 \mathrm{~s})$ & $99.02(787 \min 52 \mathrm{~s})$ & $99.32(788 \mathrm{~min} 18 \mathrm{~s})$ & $98.81(794 \min 27 \mathrm{~s})$ & $98.54(818 \mathrm{~min} 35 \mathrm{~s})$ \\
\hline & 15 & $98.77(775 \min 15 \mathrm{~s})$ & $99.02(778 \mathrm{~min} 17 \mathrm{~s})$ & $99.31(780 \mathrm{~min} 26 \mathrm{~s})$ & $98.81(761 \mathrm{~min} 39 \mathrm{~s})$ & $98.59(815 \min 29 \mathrm{~s})$ \\
\hline & 10 & $98.77(771 \mathrm{~min} 17 \mathrm{~s})$ & $99.01(773 \min 32 \mathrm{~s})$ & $99.30(776 \mathrm{~min} 09 \mathrm{~s})$ & $98.78(759 \min 05 \mathrm{~s})$ & $98.59(813 \min 16 \mathrm{~s})$ \\
\hline \multirow{5}{*}{ GoogLeNet } & 30 & $98.01(523 \min 29 \mathrm{~s})$ & $98.53(529 \min 49 \mathrm{~s})$ & $98.54(527 \min 49 \mathrm{~s})$ & $98.49(532 \min 47 \mathrm{~s})$ & $98.43(654 \min 51 \mathrm{~s})$ \\
\hline & 25 & $97.75(509 \min 17 \mathrm{~s})$ & $98.53(510 \min 24 \mathrm{~s})$ & $98.56(525 \mathrm{~min} 37 \mathrm{~s})$ & $98.48(528 \min 44 \mathrm{~s})$ & $98.45(639 \min 51 \mathrm{~s})$ \\
\hline & 20 & $97.58(501 \mathrm{~min} 22 \mathrm{~s})$ & $98.51(509 \min 17 \mathrm{~s})$ & $98.56(513 \min 18 \mathrm{~s})$ & $98.49(516 \min 15 \mathrm{~s})$ & $98.44(617 \mathrm{~min} 42 \mathrm{~s})$ \\
\hline & 15 & $97.44(498 \min 27 \mathrm{~s})$ & $98.47(506 \min 24 \mathrm{~s})$ & $98.57(507 \mathrm{~min} 09 \mathrm{~s})$ & $98.49(508 \min 27 \mathrm{~s})$ & $98.45(612 \min 33 \mathrm{~s})$ \\
\hline & 10 & $97.02(485 \min 54 \mathrm{~s})$ & $98.47(501 \mathrm{~min} 35 \mathrm{~s})$ & $98.56(503 \mathrm{~min} 38 \mathrm{~s})$ & $98.48(507 \min 44 \mathrm{~s})$ & $98.45(592 \mathrm{~min} 37 \mathrm{~s})$ \\
\hline \multirow{5}{*}{ VGG19 } & 30 & $98.49(576 \min 43 \mathrm{~s})$ & $98.49(578 \min 26 \mathrm{~s})$ & $98.49(581 \min 52 \mathrm{~s})$ & $98.48(503 \min 37 \mathrm{~s})$ & $98.41(509 \min 33 \mathrm{~s})$ \\
\hline & 25 & $98.45(558 \min 17 \mathrm{~s})$ & $98.49(566 \min 33 \mathrm{~s})$ & $98.51(577 \min 49 \mathrm{~s})$ & $98.46(496 \min 47 \mathrm{~s})$ & $98.46(504 \mathrm{~min} 18 \mathrm{~s})$ \\
\hline & 20 & $98.45(537 \mathrm{~min} 55 \mathrm{~s})$ & $98.49(541 \mathrm{~min} 34 \mathrm{~s})$ & $98.52(562 \min 22 \mathrm{~s})$ & $98.43(470 \min 29 \mathrm{~s})$ & $98.49(501 \mathrm{~min} 24 \mathrm{~s})$ \\
\hline & 15 & $98.46(528 \min 18 \mathrm{~s})$ & $98.49(527 \min 15 \mathrm{~s})$ & $98.52(512 \min 06 \mathrm{~s})$ & $98.43(451 \min 14 \mathrm{~s})$ & $98.46(489 \min 46 \mathrm{~s})$ \\
\hline & 10 & $98.44(501 \mathrm{~min} 09 \mathrm{~s})$ & $98.49(518 \min 48 \mathrm{~s})$ & $98.51(512 \min 48 \mathrm{~s})$ & $98.42(438 \min 42 \mathrm{~s})$ & $98.46(484 \min 17 \mathrm{~s})$ \\
\hline \multirow{5}{*}{ SqueezeNet } & 30 & $98.07(522 \min 24 \mathrm{~s})$ & $98.21(524 \min 45 \mathrm{~s})$ & $98.31(549 \min 42 \mathrm{~s})$ & $98.22(456 \min 16 \mathrm{~s})$ & $98.29(486 \min 19 \mathrm{~s})$ \\
\hline & 25 & $98.06(519 \min 18 \mathrm{~s})$ & $98.25(520 \mathrm{~min} 36 \mathrm{~s})$ & $98.27(540 \min 15 \mathrm{~s})$ & $98.19(441 \min 18 \mathrm{~s})$ & $98.31(483 \min 28 \mathrm{~s})$ \\
\hline & 20 & $98.07(511 \mathrm{~min} 04 \mathrm{~s})$ & $98.21(516 \min 49 \mathrm{~s})$ & $98.29(538 \min 57 \mathrm{~s})$ & $98.18(438 \min 45 \mathrm{~s})$ & $98.33(469 \min 34 \mathrm{~s})$ \\
\hline & 15 & $98.06(509 \min 19 \mathrm{~s})$ & $98.19(510 \min 39 \mathrm{~s})$ & $98.30(529 \min 42 \mathrm{~s})$ & $98.14(435 \min 57 \mathrm{~s})$ & $98.42(463 \mathrm{~min} 05 \mathrm{~s})$ \\
\hline & 10 & $98.06(501 \mathrm{~min} 31 \mathrm{~s})$ & $98.25(509 \min 24 \mathrm{~s})$ & $98.26(519 \min 16 \mathrm{~s})$ & $98.18(424 \min 19 \mathrm{~s})$ & $98.31(453 \mathrm{~min} 01 \mathrm{~s})$ \\
\hline \multirow{5}{*}{ AlexNet } & 30 & $97.71(535 \min 45 \mathrm{~s})$ & $97.73(539 \min 55 \mathrm{~s})$ & $97.77(541 \mathrm{~min} 07 \mathrm{~s})$ & $97.39(451 \min 38 \mathrm{~s})$ & $97.72(473 \min 17 \mathrm{~s})$ \\
\hline & 25 & $97.69(532 \min 44 \mathrm{~s})$ & $97.73(534 \min 49 \mathrm{~s})$ & $97.76(539 \min 33 \mathrm{~s})$ & $97.28(447 \min 42 \mathrm{~s})$ & $97.69(466 \mathrm{~min} 35 \mathrm{~s})$ \\
\hline & 20 & $97.72(532 \min 19 \mathrm{~s})$ & $97.70(533 \min 16 \mathrm{~s})$ & $97.81(539 \min 17 \mathrm{~s})$ & $97.68(443 \min 22 \mathrm{~s})$ & $97.91(457 \mathrm{~min} 08 \mathrm{~s})$ \\
\hline & 15 & $97.70(531 \mathrm{~min} 28 \mathrm{~s})$ & $97.69(531 \min 49 \mathrm{~s})$ & $97.77(534 \min 16 \mathrm{~s})$ & $97.24(441 \min 17 \mathrm{~s})$ & $97.67(453 \mathrm{~min} 03 \mathrm{~s})$ \\
\hline & 10 & $97.71(530 \mathrm{~min} 46 \mathrm{~s})$ & $97.70(531 \mathrm{~min} 04 \mathrm{~s})$ & $97.70(533 \mathrm{~min} 35 \mathrm{~s})$ & $97.23(438 \min 19 \mathrm{~s})$ & $97.67(423 \min 39 \mathrm{~s})$ \\
\hline
\end{tabular}

Bold symbols represent the maximum values of each column in the tables.

Confusion Matrix for Validation Data

\begin{tabular}{|c|c|c|c|c|c|c|c|c|c|c|}
\hline & & & & & & & & & & \\
\hline$A D I$ & 201 & & & & & & & & & $100.0 \%$ \\
\hline BACK & & 127 & & & & & & & & $100.0 \%$ \\
\hline DEB & & & 50 & & & & & & & $100.0 \%$ \\
\hline LYM & & & & 95 & & & & & & $100.0 \%$ \\
\hline MUC & & & & & 155 & & 1 & & & \begin{tabular}{|l|l|}
$99.4 \%$ & $0.6 \%$ \\
\end{tabular} \\
\hline$\frac{\pi}{0}$ MUS & & & & & & 88 & & & & $100.0 \%$ \\
\hline NORM & & & & & & & 109 & & & $100.0 \%$ \\
\hline STR & & & & & & 1 & & 63 & & \begin{tabular}{|l|l}
$98.4 \%$ & $1.6 \%$ \\
\end{tabular} \\
\hline TUM & & & 1 & & & & 1 & & 185 & \begin{tabular}{|l|l}
$98.9 \%$ & $1.1 \%$ \\
\end{tabular} \\
\hline
\end{tabular}

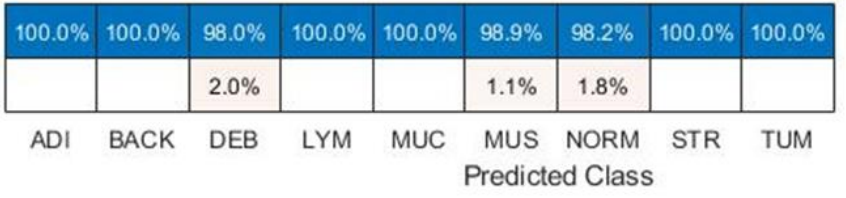

Figure 10. Experiment II: accuracy of nine classes (CRC-VAL-HE-7K). 
Table 8. Experiment II: the best result of nine tissue classes (CRC-VAL-HE-7K).

\begin{tabular}{|c|c|c|c|c|c|c|}
\hline \multicolumn{2}{|c|}{ Accuracy Rate (\%) (Times) } & \multicolumn{5}{|c|}{ Mini-Batch Size } \\
\hline Model & Epoch & 8 & 16 & 32 & 64 & 128 \\
\hline \multirow{5}{*}{ ResNet18 } & 30 & $98.03(11 \mathrm{~s})$ & $98.07(14 \mathrm{~s})$ & $98.10(16 \mathrm{~s})$ & $98.15(18 \mathrm{~s})$ & $98.08(18 \mathrm{~s})$ \\
\hline & 25 & $98.01(10 \mathrm{~s})$ & $98.07(12 \mathrm{~s})$ & $98.11(13 \mathrm{~s})$ & $98.04(16 \mathrm{~s})$ & $98.07(18 \mathrm{~s})$ \\
\hline & 20 & $97.91(7 \mathrm{~s})$ & $98.04(7 \mathrm{~s})$ & $98.20(10 \mathrm{~s})$ & 98.16 (12 s) & 97.99 (16 s) \\
\hline & 15 & $97.92(5 \mathrm{~s})$ & $97.97(6 \mathrm{~s})$ & $98.09(12 \mathrm{~s})$ & $98.16(12 \mathrm{~s})$ & 97.11 (14 s) \\
\hline & 10 & $97.91(4 \mathrm{~s})$ & $97.91(4 \mathrm{~s})$ & $98.08(8 \mathrm{~s})$ & $98.14(9 \mathrm{~s})$ & 97.08 (11 s) \\
\hline \multirow{5}{*}{ ResNet50 } & 30 & $98.56(14 \mathrm{~s})$ & 99.01 (16 s) & 99.28 (17 s) & 99.09 (19s) & $99.10(20 \mathrm{~s})$ \\
\hline & 25 & $98.53(14 \mathrm{~s})$ & 99.01 (14 s) & $99.31(15 \mathrm{~s})$ & $99.15(18 \mathrm{~s})$ & 99.17 (19s) \\
\hline & 20 & $98.51(7 \mathrm{~s})$ & $98.90(7 \mathrm{~s})$ & $99.32(9 \mathrm{~s})$ & 99.19 (12 s) & 99.09 (12 s) \\
\hline & 15 & $98.51(5 \mathrm{~s})$ & $98.88(7 \mathrm{~s})$ & $99.30(9 \mathrm{~s})$ & $99.27(10 \mathrm{~s})$ & $99.09(12 \mathrm{~s})$ \\
\hline & 10 & $98.51(5 \mathrm{~s})$ & $98.81(6 \mathrm{~s})$ & $99.30(7 \mathrm{~s})$ & $99.14(9 \mathrm{~s})$ & 99.19 (11 s) \\
\hline \multirow{5}{*}{ ResNet101 } & 30 & $98.26(15 \mathrm{~s})$ & $97.81(16 \mathrm{~s})$ & 98.07 (18 s) & $98.75(20 \mathrm{~s})$ & $98.56(20 \mathrm{~s})$ \\
\hline & 25 & $98.25(14 \mathrm{~s})$ & $97.92(15 \mathrm{~s})$ & 98.29 (16 s) & $98.71(17 \mathrm{~s})$ & $98.54(19 \mathrm{~s})$ \\
\hline & 20 & $98.29(14 \mathrm{~s})$ & $97.92(15 \mathrm{~s})$ & 98.41 (16 s) & 98.76 (17 s) & 98.33 (19s) \\
\hline & 15 & $98.31(10 \mathrm{~s})$ & $97.83(11 \mathrm{~s})$ & $98.31(12 \mathrm{~s})$ & $98.74(12 \mathrm{~s})$ & $98.19(13 \mathrm{~s})$ \\
\hline & 10 & $98.19(10 \mathrm{~s})$ & $97.90(10 \mathrm{~s})$ & 98.30 (11 s) & $98.71(12 \mathrm{~s})$ & $98.09(13 \mathrm{~s})$ \\
\hline \multirow{5}{*}{ GoogLeNet } & 30 & $97.22(7 \mathrm{~s})$ & $98.03(8 \mathrm{~s})$ & $98.04(8 \mathrm{~s})$ & $98.13(9 \mathrm{~s})$ & $98.04(11 \mathrm{~s})$ \\
\hline & 25 & $97.34(7 \mathrm{~s})$ & $98.03(7 \mathrm{~s})$ & $98.16(7 \mathrm{~s})$ & $98.17(8 \mathrm{~s})$ & $98.27(10 \mathrm{~s})$ \\
\hline & 20 & $97.29(6 \mathrm{~s})$ & $98.01(7 \mathrm{~s})$ & $98.16(7 \mathrm{~s})$ & $98.19(8 \mathrm{~s})$ & $98.23(9 \mathrm{~s})$ \\
\hline & 15 & $97.17(6 \mathrm{~s})$ & $97.97(6 \mathrm{~s})$ & $98.07(7 \mathrm{~s})$ & $98.17(7 \mathrm{~s})$ & $98.12(9 \mathrm{~s})$ \\
\hline & 10 & $97.06(6 \mathrm{~s})$ & $97.97(6 \mathrm{~s})$ & $98.06(6 \mathrm{~s})$ & 98.18 (7 s) & $98.19(7 \mathrm{~s})$ \\
\hline \multirow{5}{*}{ VGG19 } & 30 & $97.22(5 \mathrm{~s})$ & $97.32(5 \mathrm{~s})$ & $97.51(7 \mathrm{~s})$ & $97.55(7 \mathrm{~s})$ & $97.01(8 \mathrm{~s})$ \\
\hline & 25 & $97.05(4 \mathrm{~s})$ & $97.11(4 \mathrm{~s})$ & $97.47(5 \mathrm{~s})$ & $97.22(6 \mathrm{~s})$ & $97.16(6 \mathrm{~s})$ \\
\hline & 20 & 97.07 (4 s) & $97.17(5 \mathrm{~s})$ & $97.51(5 \mathrm{~s})$ & $97.29(5 \mathrm{~s})$ & $97.03(6 \mathrm{~s})$ \\
\hline & 15 & $97.19(4 \mathrm{~s})$ & $97.13(4 \mathrm{~s})$ & $97.49(4 \mathrm{~s})$ & $97.21(5 \mathrm{~s})$ & $97.14(6 \mathrm{~s})$ \\
\hline & 10 & $97.11(4 \mathrm{~s})$ & $97.14(4 \mathrm{~s})$ & $96.37(4 \mathrm{~s})$ & $97.16(4 \mathrm{~s})$ & $97.07(5 \mathrm{~s})$ \\
\hline \multirow{5}{*}{ SqueezeNet } & 30 & $96.95(8 \mathrm{~s})$ & $96.13(8 \mathrm{~s})$ & $96.09(8 \mathrm{~s})$ & $97.24(9 \mathrm{~s})$ & $97.32(11 \mathrm{~s})$ \\
\hline & 25 & $96.95(5 \mathrm{~s})$ & $96.11(6 \mathrm{~s})$ & $96.07(6 \mathrm{~s})$ & $97.17(7 \mathrm{~s})$ & $97.21(10 \mathrm{~s})$ \\
\hline & 20 & $96.91(5 \mathrm{~s})$ & $96.18(5 \mathrm{~s})$ & $96.11(6 \mathrm{~s})$ & $97.12(7 \mathrm{~s})$ & $97.40(9 \mathrm{~s})$ \\
\hline & 15 & $96.90(5 \mathrm{~s})$ & $96.17(5 \mathrm{~s})$ & $96.07(5 \mathrm{~s})$ & $97.14(7 \mathrm{~s})$ & $97.43(9 \mathrm{~s})$ \\
\hline & 10 & $96.90(4 \mathrm{~s})$ & $96.11(4 \mathrm{~s})$ & $96.01(5 \mathrm{~s})$ & $97.14(6 \mathrm{~s})$ & $97.44(8 \mathrm{~s})$ \\
\hline \multirow{5}{*}{ AlexNet } & 30 & $95.11(9 \mathrm{~s})$ & $95.27(9 \mathrm{~s})$ & $95.29(11 \mathrm{~s})$ & 95.07 (13 s) & $95.17(13 \mathrm{~s})$ \\
\hline & 25 & $95.05(9 \mathrm{~s})$ & $95.27(10 \mathrm{~s})$ & $95.28(10 \mathrm{~s})$ & 95.08 (12 s) & $95.16(14 \mathrm{~s})$ \\
\hline & 20 & $95.03(8 \mathrm{~s})$ & $95.25(10 \mathrm{~s})$ & $95.30(10 \mathrm{~s})$ & 95.07 (11 s) & 95.18 (11 s) \\
\hline & 15 & $94.97(8 \mathrm{~s})$ & $95.22(8 \mathrm{~s})$ & $95.28(9 \mathrm{~s})$ & $95.08(10 \mathrm{~s})$ & 95.18 (11 s) \\
\hline & 10 & $94.94(7 \mathrm{~s})$ & $94.25(8 \mathrm{~s})$ & $95.27(8 \mathrm{~s})$ & $95.07(10 \mathrm{~s})$ & $95.14(9 \mathrm{~s})$ \\
\hline
\end{tabular}

Bold symbols represent the maximum values of each column in the tables.

By the same token, based on the experimental results, it can be seen that, when revising the parameters, ResNet50 was found to have achieved the highest accuracy rate at 15 epoch shown in Figure 12a, and 32 mini-batch size for nine classes of CRC images, as shown in Figure 12b. Furthermore, the same parameters of the ResNet50 neural network used for eight types of CRC images achieved a ratio of $94.86 \%$ accuracy, as shown in Figure 13a,b. Further extensive experiments have been conducted to verify the efficacy of different variants of the ResNet architecture, such as ResNet18, ResNet50 and ResNet101 [26]. It is also worth noting that an accuracy rate of $99.69 \%$ can be achieved using 177 layers of a neural network (ResNet50), which is better than the $98.61 \%$ using 71 layers of ResNet 18 and the $99.31 \%$ using 347 layers of ResNet101. Furthermore, an accuracy rate of $94.86 \%$ can be achieved using 177 layers and the same parameters of a ResNet50 neural network for eight classes of CRC images, which is better than the $92.86 \%$ using 71 layers of ResNet 18 and the $94.16 \%$, using 347 layers of ResNet101. The differences between ResNet18, ResNet50 and ResNet101 are highlighted in Figure 14. It can be seen from the previous experiments 
that the best classification accuracy rate can achieved by revising the parameters and using ResNet50.

Table 9. Experiment II: the best result of eight tissue classes (Kather-texture-2016-image).

\begin{tabular}{|c|c|c|c|c|c|c|}
\hline \multicolumn{2}{|c|}{ Accuracy Rate (\%) (Times) } & \multicolumn{5}{|c|}{ Mini-Batch Size } \\
\hline Model & Epoch & 8 & 16 & 32 & 64 & 128 \\
\hline \multirow{5}{*}{ ResNet18 } & 30 & $94.12(38 \min 41 \mathrm{~s})$ & $94.14(39 \min 09 \mathrm{~s})$ & $94.16(39 \min 28 \mathrm{~s})$ & $94.18(39 \min 47 \mathrm{~s})$ & $93.24(40 \min 15 \mathrm{~s})$ \\
\hline & 25 & $94.12(37 \min 22 \mathrm{~s})$ & $94.16(37 \min 49 \mathrm{~s})$ & $94.11(38 \min 19 \mathrm{~s})$ & $94.14(38 \min 49 \mathrm{~s})$ & $93.28(39 \min 42 \mathrm{~s})$ \\
\hline & 20 & $94.11(37 \min 35 \mathrm{~s})$ & $94.16(37 \min 21 \mathrm{~s})$ & $94.15(37 \min 52 \mathrm{~s})$ & $94.16(38 \min 11 \mathrm{~s})$ & $93.29(38 \min 39 s)$ \\
\hline & 15 & $94.09(37 \min 06 \mathrm{~s})$ & $94.17(35 \mathrm{~min} 38 \mathrm{~s})$ & $94.16(36 \min 43 \mathrm{~s})$ & $94.21(36 \min 27 \mathrm{~s})$ & $93.27(37 \mathrm{~min} 54 \mathrm{~s})$ \\
\hline & 10 & $94.10(35 \min 49 \mathrm{~s})$ & $94.13(35 \mathrm{~min} 17 \mathrm{~s})$ & $94.13(36 \min 22 \mathrm{~s})$ & $94.12(36 \min 19 \mathrm{~s})$ & $93.28(36 \min 21 \mathrm{~s})$ \\
\hline \multirow{5}{*}{ ResNet50 } & 30 & $94.38(41 \mathrm{~min} 11 \mathrm{~s})$ & $94.45(41 \mathrm{~min} 35 \mathrm{~s})$ & $94.85(43 \min 41 \mathrm{~s})$ & $94.56(43 \min 52 \mathrm{~s})$ & $94.71(44 \min 29 \mathrm{~s})$ \\
\hline & 25 & $94.38(40 \min 57 \mathrm{~s})$ & $94.45(41 \mathrm{~min} 09 \mathrm{~s})$ & $94.85(42 \min 18 \mathrm{~s})$ & $94.54(43 \min 16 \mathrm{~s})$ & $94.78(44 \min 48 \mathrm{~s})$ \\
\hline & 20 & $94.35(40 \min 23 \mathrm{~s})$ & $94.43(40 \min 44 \mathrm{~s})$ & $94.86(40 \min 22 \mathrm{~s})$ & $94.56(41 \min 25 \mathrm{~s})$ & $94.72(43 \min 45 \mathrm{~s})$ \\
\hline & 15 & $94.32(39 \min 43 \mathrm{~s})$ & $94.41(40 \min 01 \mathrm{~s})$ & $94.86(40 \mathrm{~min} 18 \mathrm{~s})$ & $94.56(40 \mathrm{~min} 07 \mathrm{~s})$ & $94.79(43 \min 52 \mathrm{~s})$ \\
\hline & 10 & $94.33(39 \min 17 \mathrm{~s})$ & $94.38(39 \min 44 \mathrm{~s})$ & $94.77(40 \min 02 \mathrm{~s})$ & $94.55(40 \min 27 \mathrm{~s})$ & $94.79(43 \mathrm{~min} 31 \mathrm{~s})$ \\
\hline \multirow{5}{*}{ ResNet101 } & 30 & $92.59(41 \mathrm{~min} 32 \mathrm{~s})$ & $92.69(42 \min 54 \mathrm{~s})$ & $92.74(42 \min 57 \mathrm{~s})$ & $92.66(43 \min 17 \mathrm{~s})$ & $91.52(53 \min 41 \mathrm{~s})$ \\
\hline & 25 & $92.59(41 \min 18 \mathrm{~s})$ & $92.69(41 \min 31 \mathrm{~s})$ & $92.76(42 \min 26 \mathrm{~s})$ & $92.65(43 \min 49 \mathrm{~s})$ & $91.50(53 \mathrm{~min} 16 \mathrm{~s})$ \\
\hline & 20 & $92.55(40 \min 55 \mathrm{~s})$ & $92.71(41 \min 18 \mathrm{~s})$ & $92.76(41 \min 46 \mathrm{~s})$ & $92.66(42 \min 59 \mathrm{~s})$ & $91.49(52 \min 58 \mathrm{~s})$ \\
\hline & 15 & $92.48(40 \min 49 \mathrm{~s})$ & $92.67(41 \mathrm{~min} 01 \mathrm{~s})$ & $92.75(41 \min 36 \mathrm{~s})$ & $92.66(41 \min 43 \mathrm{~s})$ & $91.50(52 \min 19 \mathrm{~s})$ \\
\hline & 10 & $92.45(40 \min 44 \mathrm{~s})$ & $92.69(40 \min 52 \mathrm{~s})$ & $92.76(41 \mathrm{~min} 18 \mathrm{~s})$ & $92.65(41 \mathrm{~min} 37 \mathrm{~s})$ & $91.50(51 \min 47 \mathrm{~s})$ \\
\hline \multirow{5}{*}{ GoogLeNet } & 30 & $90.61(30 \min 31 \mathrm{~s})$ & $91.39(30 \min 45 \mathrm{~s})$ & $92.39(31 \mathrm{~min} 47 \mathrm{~s})$ & $92.36(31 \min 59 \mathrm{~s})$ & $92.16(31 \mathrm{~min} 30 \mathrm{~s})$ \\
\hline & 25 & $90.59(30 \min 17 \mathrm{~s})$ & $91.39(30 \min 42 \mathrm{~s})$ & $92.39(30 \min 53 \mathrm{~s})$ & $92.36(31 \min 56 \mathrm{~s})$ & $92.17(30 \min 46 \mathrm{~s})$ \\
\hline & 20 & $90.60(30 \min 01 \mathrm{~s})$ & $91.37(30 \min 19 \mathrm{~s})$ & $92.42(30 \min 17 \mathrm{~s})$ & $92.36(31 \min 44 \mathrm{~s})$ & $92.15(30 \min 17 \mathrm{~s})$ \\
\hline & 15 & $90.60(29 \min 55 \mathrm{~s})$ & $91.32(30 \min 11 \mathrm{~s})$ & $92.33(30 \min 46 \mathrm{~s})$ & $92.36(31 \min 09 \mathrm{~s})$ & $92.14(29 \min 52 \mathrm{~s})$ \\
\hline & 10 & $90.59(29 \min 34 \mathrm{~s})$ & $91.33(29 \min 54 \mathrm{~s})$ & $92.36(30 \min 34 \mathrm{~s})$ & $92.36(30 \min 54 \mathrm{~s})$ & $92.15(29 \min 29 \mathrm{~s})$ \\
\hline \multirow{5}{*}{ VGG19 } & 30 & $90.91(26 \min 29 \mathrm{~s})$ & $90.86(26 \min 17 \mathrm{~s})$ & $91.30(26 \min 17 \mathrm{~s})$ & $91.29(27 \min 21 \mathrm{~s})$ & $90.89(27 \min 29 \mathrm{~s})$ \\
\hline & 25 & $90.90(25 \min 37 \mathrm{~s})$ & $90.86(25 \min 51 \mathrm{~s})$ & $91.35(25 \min 59 \mathrm{~s})$ & $91.25(26 \min 48 \mathrm{~s})$ & $90.87(27 \mathrm{~min} 16 \mathrm{~s})$ \\
\hline & 20 & $90.91(25 \min 11 \mathrm{~s})$ & $90.85(25 \min 32 \mathrm{~s})$ & $91.37(25 \min 22 \mathrm{~s})$ & $91.29(24 \min 27 \mathrm{~s})$ & $90.89(24 \min 54 \mathrm{~s})$ \\
\hline & 15 & $90.91(24 \min 58 \mathrm{~s})$ & $90.84(25 \min 08 \mathrm{~s})$ & $91.31(25 \min 08 \mathrm{~s})$ & $91.29(24 \min 03 \mathrm{~s})$ & $90.89(24 \min 22 \mathrm{~s})$ \\
\hline & 10 & $90.89(24 \min 36 \mathrm{~s})$ & $90.84(24 \min 27 \mathrm{~s})$ & $91.32(24 \min 43 \mathrm{~s})$ & $91.27(23 \mathrm{~min} 44 \mathrm{~s})$ & $90.89(24 \min 09 \mathrm{~s})$ \\
\hline \multirow{5}{*}{ SqueezeNet } & 30 & $88.09(25 \min 27 \mathrm{~s})$ & $88.16(25 \min 44 \mathrm{~s})$ & $88.17(26 \min 14 \mathrm{~s})$ & $88.24(26 \min 47 \mathrm{~s})$ & $87.37(28 \min 49 \mathrm{~s})$ \\
\hline & 25 & $88.09(25 \min 14 \mathrm{~s})$ & $88.17(25 \min 28 \mathrm{~s})$ & $88.17(25 \min 52 \mathrm{~s})$ & $88.25(26 \min 19 \mathrm{~s})$ & $87.29(27 \min 42 \mathrm{~s})$ \\
\hline & 20 & $88.07(25 \min 02 \mathrm{~s})$ & $88.17(25 \min 25 \mathrm{~s})$ & $88.17(25 \min 44 \mathrm{~s})$ & $88.24(25 \min 58 \mathrm{~s})$ & $87.24(27 \min 19 \mathrm{~s})$ \\
\hline & 15 & $88.09(24 \min 46 \mathrm{~s})$ & $88.18(25 \min 09 \mathrm{~s})$ & $88.11(25 \min 26 \mathrm{~s})$ & $88.27(25 \min 37 \mathrm{~s})$ & $87.29(26 \min 27 \mathrm{~s})$ \\
\hline & 10 & $88.09(24 \min 25 \mathrm{~s})$ & $88.17(24 \min 49 \mathrm{~s})$ & $88.08(24 \min 38 \mathrm{~s})$ & $88.24(25 \min 06 \mathrm{~s})$ & $87.19(25 \mathrm{~min} 38 \mathrm{~s})$ \\
\hline \multirow{5}{*}{ AlexNet } & 30 & $90.18(30 \min 05 \mathrm{~s})$ & $91.94(31 \mathrm{~min} 27 \mathrm{~s})$ & $91.92(32 \min 44 \mathrm{~s})$ & $91.91(33 \min 52 \mathrm{~s})$ & $89.40(45 \min 05 \mathrm{~s})$ \\
\hline & 25 & $90.17(29 \min 45 \mathrm{~s})$ & $91.94(31 \mathrm{~min} 43 \mathrm{~s})$ & $91.94(31 \mathrm{~min} 16 \mathrm{~s})$ & $91.93(33 \min 27 \mathrm{~s})$ & $89.42(45 \min 19 \mathrm{~s})$ \\
\hline & 20 & $90.18(29 \min 33 \mathrm{~s})$ & $91.94(30 \min 22 \mathrm{~s})$ & $91.94(30 \min 41 \mathrm{~s})$ & $91.91(32 \min 41 \mathrm{~s})$ & $89.44(45 \min 37 \mathrm{~s})$ \\
\hline & 15 & $90.18(29 \min 12 \mathrm{~s})$ & $91.94(29 \min 49 \mathrm{~s})$ & $91.91(30 \min 22 \mathrm{~s})$ & $91.94(32 \min 36 \mathrm{~s})$ & $89.41(46 \min 27 \mathrm{~s})$ \\
\hline & 10 & $90.19(28 \min 58 \mathrm{~s})$ & $91.94(29 \min 23 \mathrm{~s})$ & $91.87(29 \min 27 \mathrm{~s})$ & $91.94(30 \min 11 \mathrm{~s})$ & $89.49(45 \min 44 \mathrm{~s})$ \\
\hline
\end{tabular}

Bold symbols represent the maximum values of each column in the tables.

Confusion Matrix for Validation Data

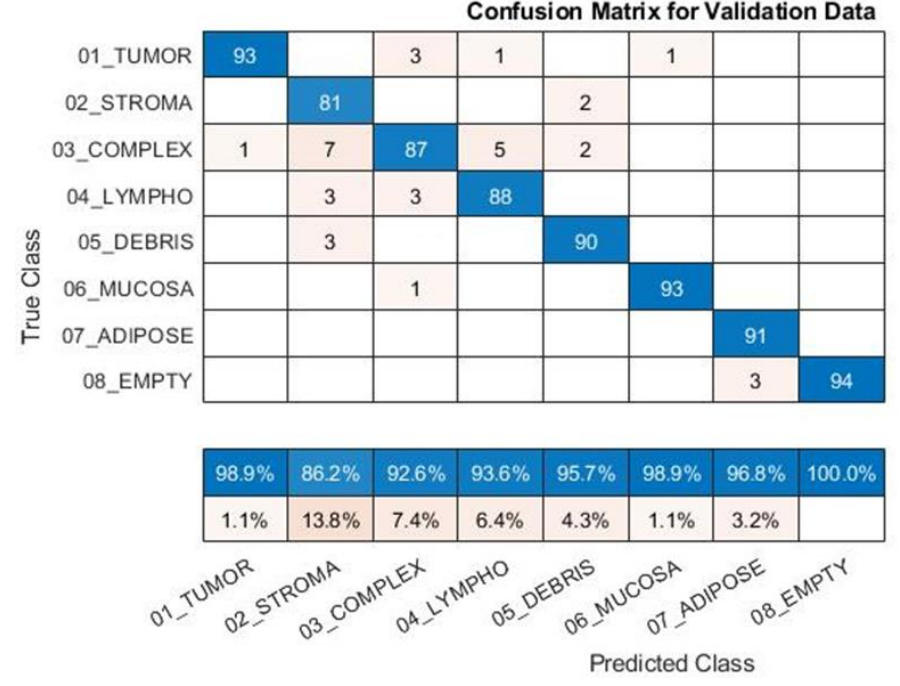

\begin{tabular}{|l|l|}
\hline $94.9 \%$ & $5.1 \%$ \\
\hline $97.6 \%$ & $2.4 \%$ \\
\hline $85.3 \%$ & $14.7 \%$ \\
\hline $93.6 \%$ & $6.4 \%$ \\
\hline $96.8 \%$ & $3.2 \%$ \\
\hline $98.9 \%$ & $1.1 \%$ \\
\hline $100.0 \%$ & \\
\hline $96.9 \%$ & $3.1 \%$ \\
\hline
\end{tabular}

Figure 11. Experiment II: accuracy of eight classes (Kather-texture-2016-image-5000). 


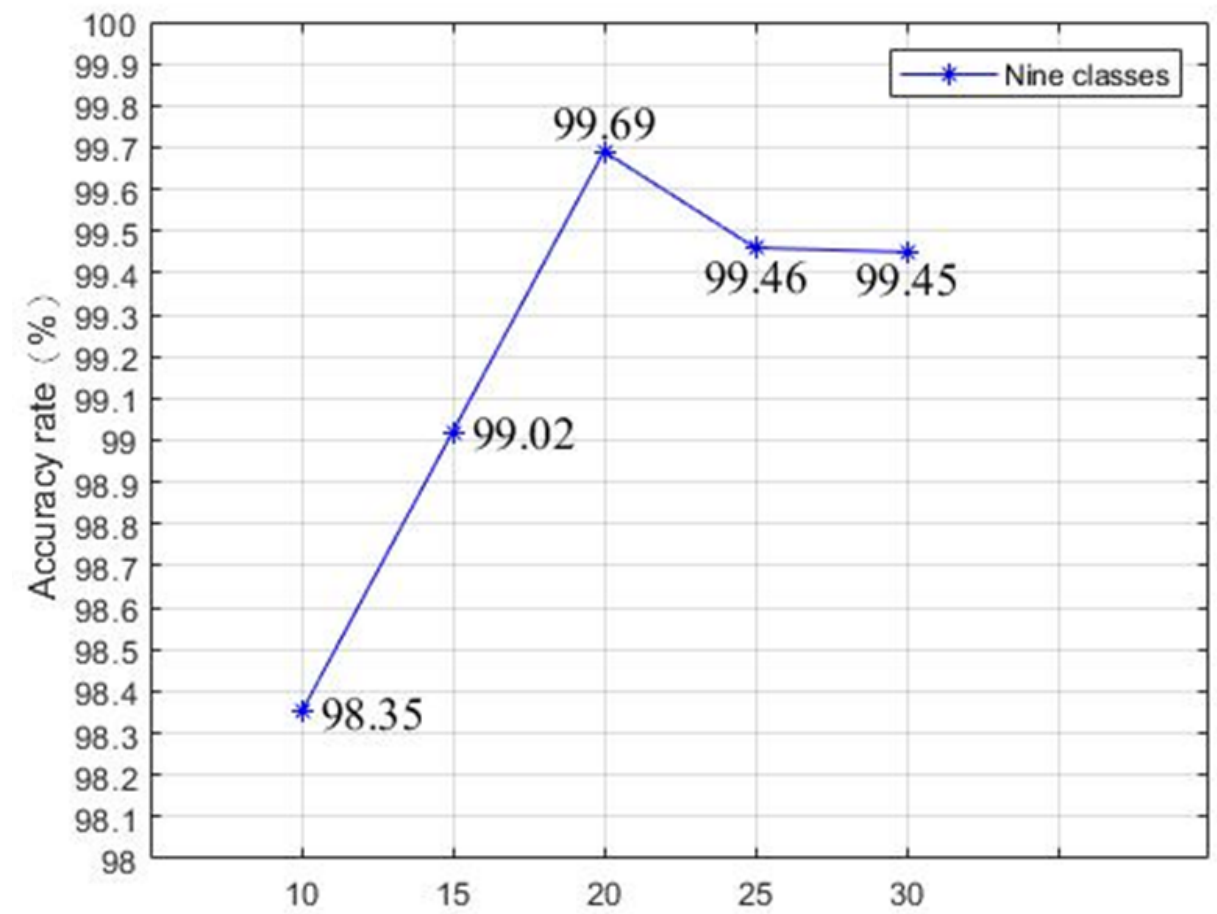

(a) Epochs

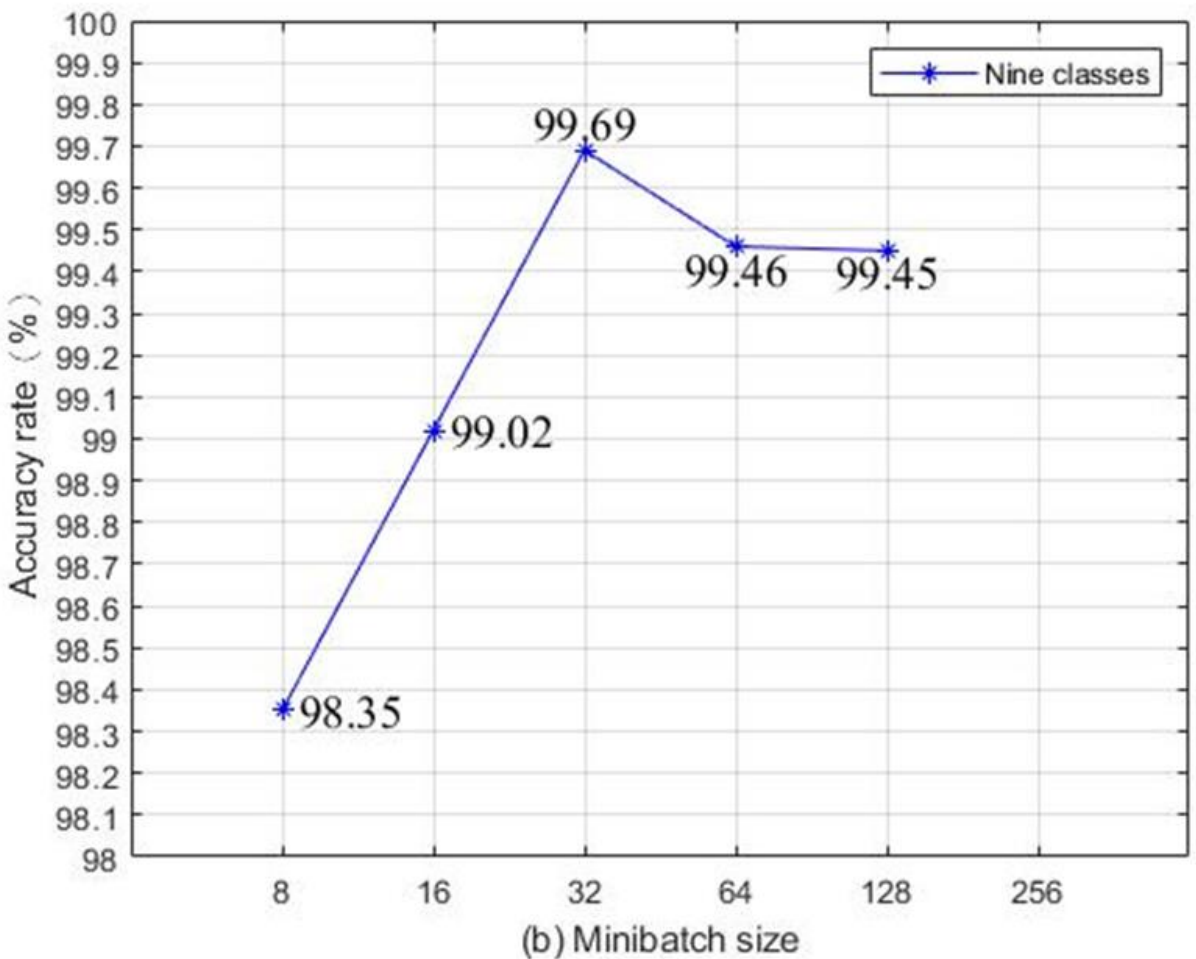

Figure 12. Illustration of the accuracy of nine tissue classes (CRC-VAL-HE-100K) using (a) epoch and (b) mini-batch size. 


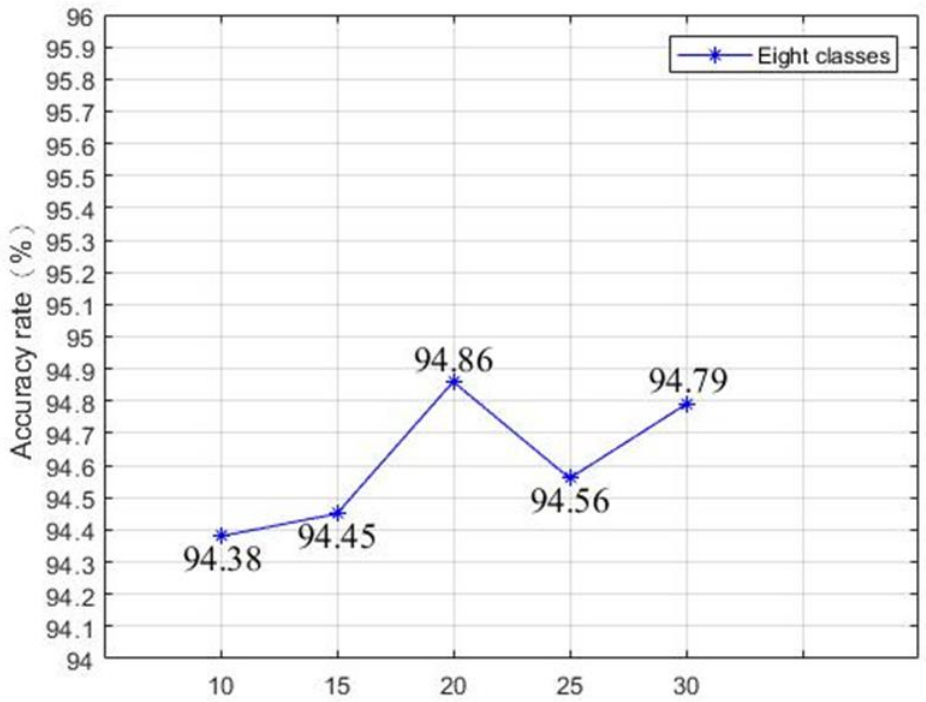

(a) Epochs

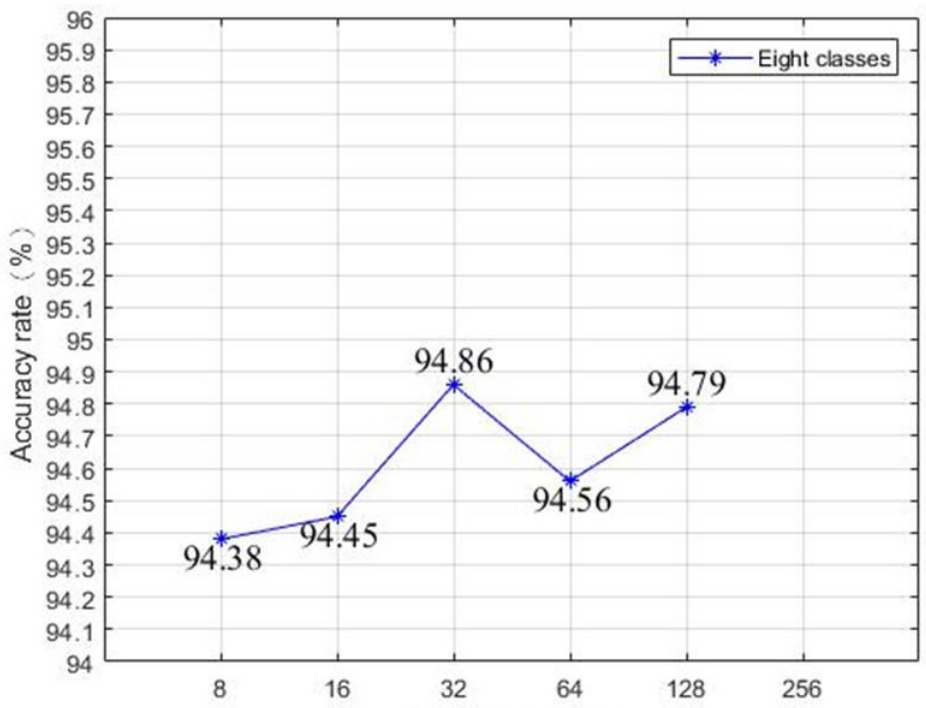

(b) Minibatch size

Figure 13. Illustration of the accuracy of eight tissue classes (Kather-texture-2016-image-5000) using (a) epoch and (b) mini-batch size.

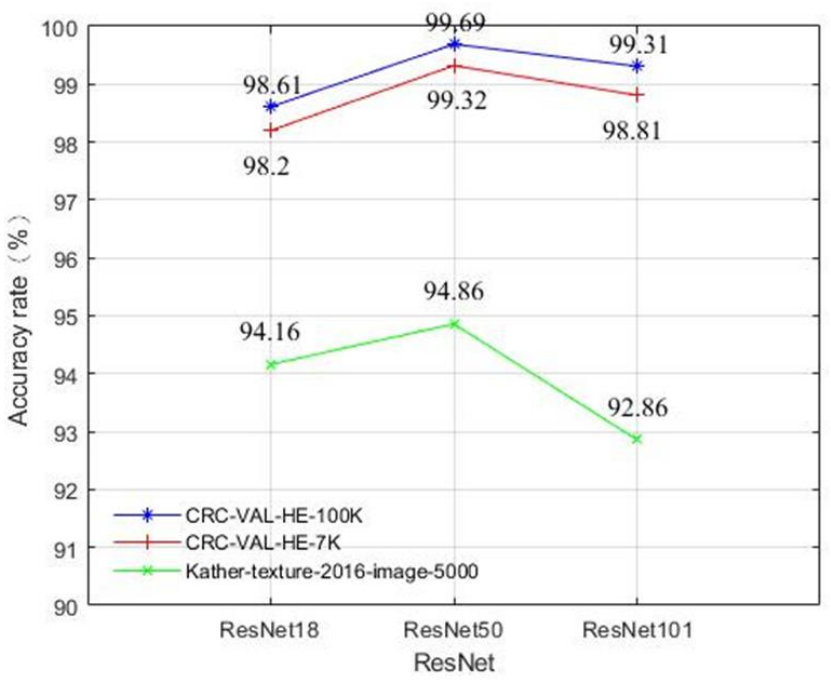

Figure 14. Accuracy rates of ResNet. 


\subsection{Discussion}

After the detailed explanation of the approach and experiments, it is necessary to compare the performance of the proposed techniques with published data. In Reference [13], Kather et al. applied the same NCT-HE-100K data set of 100,000 histological images to train a VGG19 CNN model and tested the classification performance in an independent set of 7180 images from different patients (CRC-VAL-HE-7K). The overall nine-class accuracy was close to $99 \%$ in an internal testing set and $94.3 \%$ in an external testing set. Unlike the approach in [13], the experimental results of ResNet50 outperform the data of VGG19 in Table 7. We had achieved $99.69 \%$ accuracy rate in the same internal testing set and $99.32 \%$ in the same external testing set from Figure 14. Through comprehensive and thorough analyses, this study suggests that ResNet50 could be a better deep learning architecture for colorectal cancer tissue than VGG19.

To further validate our claim, the independent data set with eight classes of [2] is also utilized for comparison purpose. Through our study, ResNet50 achieved $94.86 \%$ accuracy in Figure 14 and [2] reported the best accuracy rate was $87.4 \%$. Through comprehensive studies and comparison, it is highly suggested that ResNet50 with suggested settings of this study could be the most efficient and accuracy deep learning techniques to classify colorectal cancer tissue.

Since deep neural networks have been adopted in this study as the classifier, the modular design of those models conveniently provides their architecture to specific needs. Many factors could easily be modified like weight initialization, batch sizes, epochs, learning rates, activation function, optimizer, loss function, network topology, etc. to improve the classification accuracy. Among various settings for the superior classification performance, several studies [28-30] have suggested that loss function could be critical to affect the deep learning models and learning efficiency, as well as the classifier robustness to various situation.

In this study, the authors adopted the transfer learning of deep learning architecture for the classification of colorectal cancer tissue, those network models are optimized based on the pre-trained data from ImageNet [22]. Since ImageNet is a large labeled dataset of real-world images, it is one of the most widely used dataset in latest computer vision research and several well-known models are the ImageNet Large Scale Visual Recognition Challenge (ILSVRC) winners. The loss functions for each network adopted in this study are cross entropy loss function for all network models. The authors would pay extra attention to optimize the selection of loss function for future research in order to further improve the overall accuracy, class imbalance awareness and convergence speed for the classification of colorectal cancer tissue. Therefore, our research could effectively classify the medical images in aiding clinical care and treatment.

\section{Conclusions}

This study was based on exploring different deep learning models for the recognition of colorectal cancer tissue using CNN. An improved version of deep learning parameters was proposed in this article to improve the accuracy of classification. In order to verify our optimized parameters, we used CRC histological images as the experimental dataset, and compared the ability of the five most commonly-used deep learning network models to accurately distinguish colorectal cancer tissues. Based on the experimental results, our method was superior to the techniques described in the literature and achieved a high recognition rate. In summary, the nine-class accuracy of NCT-HE-100K data set of 100,000 histological images was close to $99 \%$ in an internal testing set and $94.3 \%$ in an external testing set in [3]. However, the experimental results of ResNet50 in this study achieved $99.69 \%$ accuracy rate in the same internal testing set and $99.32 \%$ in the same external testing set which outperform the data of VGG19 of [3]. In addition, the independent data set with eight classes of [2] is also utilized for comparison purpose. Consequently, ResNet50 achieved $94.86 \%$ accuracy and [2] reported the best accuracy rate was $87.4 \%$. Through comprehensive studies and comparison, it is highly suggested that ResNet50 with 
suggested settings of this study could be the most efficient and accuracy deep learning techniques to classify colorectal cancer tissue.

In short, the experimental results demonstrate that artificial intelligence has a broad application in classifying colorectal cancer (CRC) histology images, and it can also enhance doctors' critical thinking skills and enable them to make suitable decisions in the diagnostic process.

Author Contributions: Data curation, Y.-H.T.; writing-review \& editing, M.-J.T. All authors have read and agreed to the published version of the manuscript.

Funding: This work was partially supported by the National Science Council in Taiwan, Republic of China, grant number MOST 109-2410-H-009-022-MY3.

Acknowledgments: The authors thank to National Center for High-performance Computing (NCHC) of National Applied Research Laboratories (NARLabs) in Taiwan for providing computational and storage resources.

Conflicts of Interest: The authors declare no conflict of interest.

\section{References}

1. Egeblad, M.; Nakasone, E.S.; Werb, Z. Tumors as Organs: Complex Tissues that Interface with the Entire Organism. Dev. Cell 2010, 18, 884-901. [CrossRef] [PubMed]

2. Kather, J.N.; Weis, C.-A.; Bianconi, F.; Melchers, S.M.; Schad, L.R.; Gaiser, T.; Marx, A.; Zöllner, F. Multi-class texture analysis in colorectal cancer histology. Sci. Rep. 2016, 6, 27988. [CrossRef]

3. Kather, J.N.; Krisam, J.; Charoentong, P.; Luedde, T.; Herpel, E.; Weis, C.-A.; Gaiser, T.; Marx, A.; Valous, N.A.; Ferber, D.; et al. Predicting survival from colorectal cancer histology slides using deep learning: A retrospective multicenter study. PLoS Med. 2019, 16, e1002730. [CrossRef]

4. Gurcan, M.N.; Boucheron, L.E.; Can, A.; Madabhushi, A.; Rajpoot, N.M.; Yener, B. Histopathological Image Analysis: A Review. IEEE Rev. Biomed. Eng. 2009, 2, 147-171. [CrossRef] [PubMed]

5. Zhang, S.; Metaxas, D. Large-scale medical image analytics: Recent methodologies, applications and future directions. Med. Image Anal. 2016, 33, 98-101. [CrossRef] [PubMed]

6. Zhang, X.; Su, H.; Yang, L.; Zhang, S. Fine-grained histopathological image analysis via robust segmentation and large-scale retrieval. In Proceedings of the IEEE Conference on Computer Vision and Pattern Recognition (CVPR), Boston, MA, USA, 7-12 June 2015; pp. 5361-5368.

7. Janowczyk, A.; Madabhushi, A. Deep learning for digital pathology image analysis: A comprehensive tutorial with selected use cases. J. Pathol. Inform. 2016, 7, 29. [CrossRef]

8. Korbar, B.; Olofson, A.M.; Miraflor, A.P.; Nicka, C.M.; Suriawinata, M.A.; Torresani, L.; Suriawinata, A.A.; Hassanpour, S. Deep learning for classification of colorectal polyps on whole-slide images. J. Pathol. Inform. 2017, 8. [CrossRef]

9. Tsai, M.J.; Tao, Y.H. Machine Learning Based Common Radiologist-Level Pneumonia Detection on Chest X-rays. In Proceedings of the 2019 13th International Conference on Signal Processing and Communication Systems (ICSPCS), Gold Coast, Australia, 16-18 December 2019.

10. Xu, J.; Luo, X.; Wang, G.; Gilmore, H.; Madabhushi, A. A Deep Convolutional Neural Network for segmenting and classifying epithelial and stromal regions in histopathological images. Neurocomputing 2016, 191, 214-223. [CrossRef]

11. Du, Y.; Zhang, R.; Zargari, A.; Thai, T.C.; Gunderson, C.C.; Moxley, K.M.; Liu, H.; Zheng, B.; Qiu, Y. Classification of tumor epithelium and stroma by exploiting image features learned by deep convolutional neural networks. Ann. Biomed. Eng. 2018, 46, 1988-1999. [CrossRef]

12. Xu, Y.; Jia, Z.; Wang, L.-B.; Ai, Y.; Zhang, F.; Lai, M.; Chang, E.I.-C. Large scale tissue histopathology image classification, segmentation, and visualization via deep convolutional activation features. BMC Bioinform. 2017, 18, 281. [CrossRef]

13. Bejnordi, B.E.; Mullooly, M.; Pfeiffer, R.M.; Fan, S.; Vacek, P.M.; Weaver, D.L.; Herschorn, S.; Brinton, L.A.; Van Ginneken, B.; Karssemeijer, N.; et al. Using deep convolutional neural networks to identify and classify tumor-associated stroma in diagnostic breast biopsies. Mod. Pathol. 2018, 31, 1502-1512. [CrossRef]

14. Bowles, M. Machine Learning in Python: Essential Techniques for Predictive Analysis; John Wiley \& Sons: Hoboken, NJ, USA, 2015.

15. Hubel, D.H.; Wiesel, T.N. Receptive fields, binocular interaction and functional architecture in the cat's visual cortex. J. Physiol. 1962, 160, 106-154. [CrossRef]

16. Hinton, G.; Salakhutdinov, R. Reducing the Dimensionality of Data with Neural Networks. Science 2006, 313, 504-507. [CrossRef]

17. Markoff, J. How Many Computers to Identify a Cat. 22 June 2012. Available online: https:/ / mobile.nytimes.com/2012/06/26 /technology/in-a-big-network-of-computers-evidence-of-machine-learning.html (accessed on 11 July 2020).

18. LeCun, Y.; Bottou, L.; Bengio, Y.; Haffner, P. Gradient-Based Learning Applied to Document Recognition. Proc. IEEE 1998, 86, 2278-2324. [CrossRef]

19. Lin, M.; Chen, Q.; Yan, S. Network in Network, Cornell University Library. arXiv 2014, arXiv:1312.4400. 
20. Rumelhart, E.; Geoffrey, E.; Ronald, J. Learning representations by back-propagating errors. Nature 1986, 323, 533-536. [CrossRef]

21. Yadav, S.S.; Jadhav, S.M. Deep convolutional neural network based medical image classification for disease diagnosis. J. Big Data 2019, 6, 1-18. [CrossRef]

22. Krizhevsky, A.; Sutskever, I.; Hinton, G.E. ImageNet classification with deep convolutional neural networks. In Proceedings of the International Conference on Neural Information Processing Systems, Lake Tahoe, NV, USA, 3-6 December 2012; pp. 1097-1105.

23. Iandola, F.N.; Han, S.; Moskewicz, M.W.; Ashraf, K.; Dally, W.J.; Keutzer, K. SqueezeNet: AlexNet-level accuracy with 50x fewer parameters and $<0.5 \mathrm{MB}$ model size. arXiv 2016, arXiv:1602.07360.

24. Simonyan, K.Z. Very Deep Convolutional Networks for Large-Scale Image Recognition. arXiv 2014, arXiv:1409.1556.

25. Szegedy, C.; Liu, W.; Jia, Y.; Sermanet, P.; Reed, S.; Anguelov, D.; Erhan, D.; Vanhoucke, V.; Rabinovich, A. Going deeper with convolutions. In Proceedings of the IEEE Conference on Computer Vision and Pattern Recognition (CVPR), Boston, MA, USA, 7-12 June 2015; pp. 7-12.

26. He, K.; Zhang, X.; Ren, S.; Sun, J. Deep residual learning for image recognition. In Proceedings of the IEEE Conference on Computer Vision and Pattern Recognition, Seattle, WA, USA, 1 June 2016; pp. 770-778.

27. Yaqub, M.; Feng, J.; Zia, M.; Arshid, K.; Jia, K.; Rehman, Z.; Mehmood, A. State-of-the-Art CNN Optimizer for Brain Tumor Segmentation in Magnetic Resonance Images. Brain Sci. 2020, 10, 427. [CrossRef] [PubMed]

28. Janocha, K.; Czarnecki, W.M. On Loss Functions for Deep Neural Networks in Classification. Schedae Inform. 2016, 25, 49-59. [CrossRef]

29. Manwar, R.; Li, X.; Mahmoodkalayeh, S.; Asano, E.; Zhu, D.; Avanaki, K. Deep learning protocol for improved photoacoustic brain imaging. J. Biophotonics 2020, 13, e202000212. [CrossRef] [PubMed]

30. Yessou, H.; Sumbul, G.; Demir, B. A Comparative Study of Deep Learning Loss Functions for Multi-Label Remote Sensing Image Classification. In Proceedings of the IGARSS 2020-2020 IEEE International Geoscience and Remote Sensing Symposium, Waikoloa, HI, USA, 26 September-2 October 2020. [CrossRef] 